$11-1-2013$

\title{
Generalized Modified Ratio Estimator for Estimation of Finite Population Mean
}

Jambulingam Subramani

PondicherryUniversity, Puducherry, India, drjsubramani@yahoo.co.in

Follow this and additional works at: http://digitalcommons.wayne.edu/jmasm

Part of the Applied Statistics Commons, Social and Behavioral Sciences Commons, and the Statistical Theory Commons

\section{Recommended Citation}

Subramani, Jambulingam (2013) "Generalized Modified Ratio Estimator for Estimation of Finite Population Mean," Journal of Modern Applied Statistical Methods: Vol. 12 : Iss. 2 , Article 7.

DOI: $10.22237 /$ jmasm/1383278760

Available at: http://digitalcommons.wayne.edu/jmasm/vol12/iss2/7

This Regular Article is brought to you for free and open access by the Open Access Journals at DigitalCommons@WayneState. It has been accepted for inclusion in Journal of Modern Applied Statistical Methods by an authorized editor of DigitalCommons@WayneState. 


\section{Generalized Modified Ratio Estimator for Estimation of Finite Population Mean}

\section{Cover Page Footnote}

The author wishes to record his gratitude and thanks to University Grants Commission (UGC) for the financial assistance through UGC-Major Research Project. 


\title{
Generalized Modified Ratio Estimator for Estimation of Finite Population Mean
}

\author{
Jambulingam Subramani \\ Pondicherry University \\ Puducherry, India
}

\begin{abstract}
A generalized modified ratio estimator is proposed for estimating the population mean using the known population parameters. It is shown that the simple random sampling without replacement sample mean, the usual ratio estimator, the linear regression estimator and all the existing modified ratio estimators are the particular cases of the proposed estimator. The bias and the mean squared error of the proposed estimator are derived and are compared with that of existing estimators. The conditions for which the proposed estimator performs better than the existing estimators are also derived. The performance of the proposed estimator is assessed with that of the existing estimators for certain natural populations
\end{abstract}

Keywords: $\quad$ Auxiliary variable, biases, natural population, mean squared error, parameters

\section{Introduction}

Consider a finite population $U=\left\{U_{1}, U_{2}, \ldots, U_{N}\right\}$ of $N$ distinct and identifiable units. Let $Y$ be a study variable with value $Y_{i}$ measured on $U_{1}, i=1,2,3, \ldots, N$ giving a vector $Y=\left\{Y_{1}, Y_{2}, \ldots, Y_{N}\right\}$. The problem is to estimate the population mean $\bar{Y}=\frac{1}{N} \sum_{i=1}^{N} Y_{i}$ on the basis of a random sample selected from the population $U$. The simple random sample mean is the simplest estimator for estimating the population mean. If an auxiliary variable $X$, closely related to the study variable $Y$, is available then one can improve the performance of the estimator of the study variable by using the known values of the population parameters of the auxiliary variable. That is, when the population parameters of the auxiliary variable $X$ such as population mean, coefficient of variation, coefficient of kurtosis, coefficient of skewness etc., are known, then a number of estimators available in the literature (such as ratio, product and linear regression

Dr. Subramani is Associate Professor and Head of the Department of Statistics. Email himat:drjsubramani@yahoo.co.in. 


\section{MODIFIED RATIO FOR ESTIMATION OF FINITE POPULATION MEAN}

estimators and their modifications) perform better than the usual simple random sample mean under certain conditions. Among these estimators, many researchers have used the ratio estimator and its modifications for the estimation of the mean of the study variable (see for example Sisodia and Dwivedi (1981), Kadilar and Cingi (2006a, 2006b), Yan and Tian (2010) and Subramani and Kumarapandiyan (2012a, 2012c)). Before discussing further the existing estimators and the proposed estimators, the notations to be used in this article are described below:

$\begin{array}{ll}N & \text { Population size } \\ n & \text { Sample size } \\ f=n / N & \text { Sampling fraction } \\ Y & \text { Study variable } \\ X & \text { Auxiliary variable } \\ \bar{X}, \bar{Y} & \text { Population means } \\ \bar{x}, \bar{y} & \text { Sample means } \\ S_{x}, S_{y} & \text { Population standard deviations } \\ C_{x}, C_{y} & \text { Co-efficient of variations } \\ \rho & \text { Co-efficient of correlation between } X \text { and } Y \\ \beta_{1} & \text { Co-efficient of skewness of the auxiliary variable } \\ \beta_{2} & \text { Co-efficient of kurtosis of the auxiliary variable } \\ M_{d} & \text { Median of the auxiliary variable } \\ B(.) & \text { Bias of the estimator } \\ M S E(.) & \text { Mean squared error of the estimator } \\ \hat{\bar{Y}}_{i}\left(\hat{\bar{Y}}_{p_{j}}\right) & \end{array}$

In case of simple random sampling without replacement (SRSWOR), the sample mean $\bar{y}_{\text {srs }}$ is used to estimate population mean $\bar{Y}$, which is an unbiased estimator, and its variance is given below:

$$
V\left(\bar{y}_{s r s}\right)=\frac{(1-f)}{n} S_{y}^{2}
$$

The ratio estimator for estimating the population mean $\bar{Y}$ of the study variable $Y$ is defined as: 


\section{JAMBULINGAM SUBRAMANI}

$$
\hat{\overline{Y_{R}}}=\frac{\bar{y}}{\bar{X}} \bar{X}=\hat{R} \bar{X} \text { where } \hat{R}=\frac{\bar{y}}{\bar{X}}=\frac{y}{x}
$$

The bias and mean squared error of the ratio estimator to the first degree of approximation are given below:

$$
\begin{gathered}
B(\hat{\bar{Y}})=\frac{(1-f)}{n} \bar{Y}\left(C_{x}^{2}-\rho C_{x} C_{y}\right) \\
\operatorname{MSE}\left(\hat{\overline{Y_{R}}}\right)=\frac{(1-f)}{n} \bar{Y}^{2}\left(C_{y}^{2}+C_{x}^{2}-2 \rho C_{x} C_{y}\right)
\end{gathered}
$$

The usual linear regression estimator together with its variance is given below:

$$
\begin{gathered}
\hat{\overline{Y_{l}}}=\bar{y}+\beta(\bar{X}-\bar{x}) \\
V\left(\hat{\overline{Y_{l r}}}\right)=\frac{(1-f)}{n} S_{y}^{2}\left(1-\rho^{2}\right)
\end{gathered}
$$

Sisodia and Dwivedi (1981) have suggested a modified ratio estimator using the co-efficient of variation of auxiliary variable $X$ for estimating $\bar{Y}$. When the coefficient of kurtosis of auxiliary variable $X$ is known, Singh et al. (2004) has developed a modified ratio estimator. Singh and Tailor (2003) proposed another estimator for estimating $\bar{Y}$ when the population correlation co-efficient between $X$ and $Y$ is known. By using the population variance of auxiliary variable $X$, Singh (2003) proposed another modified ratio estimator for estimating population mean. More recently, Yan and Tian (2010) has suggested another modified ratio estimator using the co-efficient of skewness of the auxiliary variable $X$, and Subramani and Kumarapandiyan (2013a) suggested a new modified ratio estimator using known population median of auxiliary variable $X$.

Upadhyaya and Singh (1999) suggested another modified ratio estimator using the linear combination of co-efficient of variation and co-efficient of kurtosis. Singh (2003) used the linear combination of co-efficient of kurtosis and standard deviation and co-efficient of skewness and standard deviation for estimating the populations mean $\bar{Y}$. Motivated by Singh (2003), Yan and Tian (2010) used the linear combination of co-efficient of kurtosis and co-efficient of 


\section{MODIFIED RATIO FOR ESTIMATION OF FINITE POPULATION MEAN}

skewness and co-efficient of variation and co-efficient of skewness. Subramani and Kumarapandiyan (2012a, 2012b, 2012c and 2013b) suggested modified ratio estimators using known median and co-efficient of kurtosis, median and coefficient of skewness, median and co-efficient of variation and median and coefficient of correlation.

More detailed discussion about the ratio estimator and its modification can be found in Abdia and Shahbaz (2006), Ahmad et al. (2009), Al-Jararha and AlHaj Ebrahem (2012), Bhushan (2012), Cochran (1977), Dalabehera and Sahoo (1994), David and Sukhatme (1974), Goodman and Hartley (1958), Gupta and Shabbir (2008), Jhajj et al. (2006), Kadilar and Cingi (2003, 2004), Khoshnevisan et al. (2007), Koyuncu and Kadilar (2009), Kulkarni (1978), Murthy (1967), Naik and Gupta (1991), Olkin (1958), Pathak (1964), Perri (2007), Ray and Sahai (1980), Reddy (1973), Robinson (1987), Sen (1993), Shabbir and Yaab (2003), Sharma and Tailor (2010), Singh and Chaudhary (1986), Singh (2003), Singh and Espejo (2003), Singh and Agnihotri (2008), Singh and Solanki (2012), Singh and Tailor (2003, 2005), Singh et al. (2004, 2008), Sisodia and Dwivedi (1981), Solanki et al. (2012), Srivenkataramana (1980), Tailor and Sharma (2009), Tin (1965), Upadhyaya and Singh (1999) and Yan and Tian (2010).

The following table contains all modified ratio estimators using known population parameters of the auxiliary variable in which some of the estimators are already suggested in the literature. The remaining estimators are introduced in this article:

Table 1. Modified Ratio estimators with the constant, the bias, and the mean squared errors.

\begin{tabular}{llll}
\hline Estimator & Constant $\boldsymbol{\theta}_{\boldsymbol{i}}$ & Bias $-\boldsymbol{B}()$. & Mean squared error MSE(.) \\
\hline $\begin{array}{l}\hat{\bar{Y}}=\bar{y}\left[\frac{\bar{X}+C_{x}}{\bar{x}+C_{x}}\right] \\
\begin{array}{l}\text { Sisodia and } \\
\text { Dwivedi }(1981)\end{array}\end{array}$ & $\theta_{1}=\frac{\bar{X}}{\bar{X}+C_{x}}$ & $\frac{(1-f)}{n} \bar{Y}\left(\theta_{1}^{2} C_{x}^{2}-\theta_{1} \rho C_{x} C_{y}\right)$ & $\frac{(1-f)}{n} \bar{Y}^{2}\left(C_{y}^{2}+\theta_{1}^{2} C_{x}^{2}-2 \theta_{1} \rho C_{x} C_{y}\right)$ \\
\hline $\begin{array}{l}\hat{\bar{Y}}_{2}=\bar{y}\left[\frac{\bar{X}+\beta_{2}}{\bar{X}+\beta_{2}}\right] \\
\text { Singh et al. (2004) }\end{array}$ & $\theta_{2}=\frac{\bar{X}}{\bar{X}+\beta_{2}}$ & $\frac{(1-f)}{n} \bar{Y}\left(\theta_{2}^{2} C_{x}^{2}-\theta_{2} \rho C_{x} C_{y}\right)$ & $\frac{(1-f)}{n} \bar{Y}^{2}\left(C_{y}^{2}+\theta_{2}^{2} C_{x}^{2}-2 \theta_{2} \rho C_{x} C_{y}\right)$ \\
\hline $\begin{array}{l}\hat{\bar{Y}}_{3}=\bar{y}\left[\overline{\bar{X}}+\beta_{1}\right] \\
\text { Yan and Tian } \\
(2010)\end{array}$ & $\theta_{3}=\frac{\bar{X}}{\bar{X}+\beta_{1}}$ & $\frac{(1-f)}{n} \bar{Y}\left(\theta_{3}^{2} C_{x}^{2}-\theta_{3} \rho C_{x} C_{y}\right)$ & $\frac{(1-f)}{n} \bar{Y}^{2}\left(C_{y}^{2}+\theta_{3}^{2} C_{x}^{2}-2 \theta_{3} \rho C_{x} C_{y}\right)$ \\
\hline $\begin{array}{l}\hat{\bar{Y}}_{4}=\bar{y}\left[\frac{\bar{X}+\rho}{\bar{x}+\rho}\right] \\
\begin{array}{l}\text { Singh and Tailor } \\
(2003)\end{array}\end{array}$ & $\theta_{4}=\frac{\bar{X}}{\bar{X}+\rho}$ & $\frac{(1-f)}{n} \bar{Y}\left(\theta_{4}^{2} C_{x}^{2}-\theta_{4} \rho C_{x} C_{y}\right)$ & $\frac{(1-f)}{n} \bar{Y}^{2}\left(C_{y}^{2}+\theta_{4}^{2} C_{x}^{2}-2 \theta_{4} \rho C_{x} C_{y}\right)$ \\
\hline
\end{tabular}




\section{JAMBULINGAM SUBRAMANI}

\section{Table 1 Continued}

\begin{tabular}{|c|c|c|c|}
\hline Estimator & Constant $\theta_{i}$ & Bias - $B()$. & Mean squared error MSE(.) \\
\hline $\begin{array}{l}\hat{\bar{Y}}_{5}=\bar{y}\left[\overline{\bar{X}+S_{x}} \overline{\bar{x}+S_{x}}\right] \\
\operatorname{Singh}(2003)\end{array}$ & $\theta_{5}=\frac{\bar{X}}{\bar{X}+S_{x}}$ & $\frac{(1-f)}{n} \bar{Y}\left(\theta_{5}^{2} C_{x}^{2}-\theta_{5} \rho C_{x} C_{y}\right)$ & $\frac{(1-f)}{n} \bar{Y}^{2}\left(C_{y}^{2}+\theta_{5}^{2} C_{x}^{2}-2 \theta_{5} \rho C_{x} C_{y}\right)$ \\
\hline $\begin{array}{l}\hat{\bar{Y}}_{\mathrm{v}}=\bar{y}\left[\frac{\bar{y}+M_{d}}{\bar{x}+M_{d}}\right] \\
\text { Subramani and } \\
\text { Kumarapandiyan } \\
\text { (2013a) }\end{array}$ & $\theta_{6}=\frac{\bar{X}}{\bar{X}+M_{d}}$ & $\frac{(1-f)}{n} \bar{Y}\left(\theta_{6}^{2} C_{x}^{2}-\theta_{6} \rho C_{x} C_{y}\right)$ & $\frac{(1-f)}{n} \bar{Y}^{2}\left(C_{y}^{2}+\theta_{6}^{2} C_{x}^{2}-2 \theta_{6} \rho C_{x} C_{y}\right)$ \\
\hline $\begin{array}{l}\hat{\bar{y}}_{=}=\bar{y}\left[\frac{\beta_{2} \bar{X}+C_{x}}{\beta_{2} \bar{x}+C_{x}}\right] \\
\text { Upadhyaya and } \\
\text { Singh (1999) }\end{array}$ & $\theta_{7}=\frac{\beta_{2} \bar{X}}{\beta_{2} \bar{X}+C_{x}}$ & $\frac{(1-f)}{n} \bar{Y}\left(\theta_{7}^{2} C_{x}^{2}-\theta_{7} \rho C_{x} C_{y}\right)$ & $\frac{(1-f)}{n} \bar{Y}^{2}\left(C_{y}^{2}+\theta_{7}^{2} C_{x}^{2}-2 \theta_{7} \rho C_{x} C_{y}\right)$ \\
\hline $\begin{array}{l}\hat{\bar{y}}_{2}=\bar{y}\left[\frac{C_{2} \bar{X}+\beta_{2}}{C_{1} \bar{x}+\beta_{2}}\right] \\
\text { Upadhyaya and } \\
\text { Singh (1999) }\end{array}$ & $\theta_{8}=\frac{C_{x} \bar{X}}{C_{x} \bar{X}+\beta_{2}}$ & $\frac{(1-f)}{n} \bar{Y}\left(\theta_{8}^{2} C_{x}^{2}-\theta_{8} \rho C_{x} C_{y}\right)$ & $\frac{(1-f)}{n} \bar{Y}^{2}\left(C_{y}^{2}+\theta_{8}^{2} C_{x}^{2}-2 \theta_{8} \rho C_{x} C_{y}\right)$ \\
\hline$\hat{\bar{Y}}_{9}=\bar{y}\left[\frac{\beta_{1} \bar{X}+C_{x}}{\beta_{1} \bar{X}+C_{x}}\right]$ & $\theta_{9}=\frac{\beta_{1} \bar{X}}{\beta_{1} \bar{X}+C_{x}}$ & $\frac{(1-f)}{n} \bar{Y}\left(\theta_{9}^{2} C_{x}^{2}-\theta_{9} \rho C_{x} C_{y}\right)$ & $\frac{(1-f)}{n} \bar{Y}^{2}\left(C_{y}^{2}+\theta_{9}^{2} C_{x}^{2}-2 \theta_{9} \rho C_{x} C_{y}\right)$ \\
\hline $\begin{array}{l}\hat{\bar{Y}}_{10}=\bar{y}\left[\frac{C_{X} \bar{x}+\beta_{1}}{C_{\bar{X}} \bar{x}+\beta_{1}}\right] \\
\text { Yan and Tian } \\
\text { (2010) }\end{array}$ & $\theta_{10}=\frac{C_{x} \bar{X}}{C_{x} \bar{X}+\beta_{1}}$ & $\frac{(1-f)}{n} \bar{Y}\left(\theta_{10}^{2} C_{x}^{2}-\theta_{10} \rho C_{x} C_{y}\right)$ & $\frac{(1-f)}{n} \bar{Y}^{2}\left(C_{y}^{2}+\theta_{10}^{2} C_{x}^{2}-2 \theta_{10} \rho C_{x} C_{y}\right)$ \\
\hline$\hat{\bar{Y}}_{11}=\bar{y}\left[\frac{\rho \bar{X}+C_{x}}{\rho \bar{x}+C_{x}}\right]$ & $\theta_{11}=\frac{\rho \bar{X}}{\rho \bar{X}+C_{x}}$ & $\frac{(1-f)}{n} \bar{Y}\left(\theta_{11}^{2} C_{x}^{2}-\theta_{11} \rho C_{x} C_{y}\right)$ & $\frac{(1-f)}{n} \bar{Y}^{2}\left(C_{y}^{2}+\theta_{11}^{2} C_{x}^{2}-2 \theta_{11} \rho C_{x} C_{y}\right)$ \\
\hline$\hat{\bar{Y}}_{12}=\bar{y}\left[\frac{C_{x} \bar{X}+\rho}{C_{x} \bar{x}+\rho}\right]$ & $\theta_{12}=\frac{C_{x} \bar{X}}{C_{x} \bar{X}+\rho}$ & $\frac{(1-f)}{n} \bar{Y}\left(\theta_{12}^{2} C_{x}^{2}-\theta_{12} \rho C_{x} C_{y}\right)$ & $\frac{(1-f)}{n} \bar{Y}^{2}\left(C_{y}^{2}+\theta_{12}^{2} C_{x}^{2}-2 \theta_{12} \rho C_{x} C_{y}\right)$ \\
\hline$\hat{\bar{Y}}_{13}=\bar{y}\left[\frac{S_{x} \bar{X}+C_{x}}{S_{x} \bar{x}+C_{x}}\right]$ & $\theta_{13}=\frac{S_{x} \bar{X}}{S_{x} \bar{X}+C_{x}}$ & $\frac{(1-f)}{n} \bar{Y}\left(\theta_{13}^{2} C_{x}^{2}-\theta_{13} \rho C_{x} C_{y}\right)$ & $\frac{(1-f)}{n} \bar{Y}^{2}\left(C_{y}^{2}+\theta_{11}^{2} C_{x}^{2}-2 \theta_{11} \rho C_{x} C_{y}\right)$ \\
\hline$\hat{\bar{Y}}_{14}=\bar{y}\left[\frac{C_{x} \bar{X}+S_{x}}{C_{x} \bar{x}+S_{x}}\right]$ & $\theta_{14}=\frac{C_{x} \bar{X}}{C_{x} \bar{X}+S_{x}}$ & $\frac{(1-f)}{n} \bar{Y}\left(\theta_{14}^{2} C_{x}^{2}-\theta_{14} \rho C_{x} C_{y}\right)$ & $\frac{(1-f)}{n} \bar{Y}^{2}\left(C_{y}^{2}+\theta_{14}^{2} C_{x}^{2}-2 \theta_{14} \rho C_{x} C_{y}\right)$ \\
\hline$\hat{\hat{Y}}_{15}=\bar{y}\left[\frac{M_{d} \bar{X}+C_{x}}{M_{d} \bar{x}+C_{x}}\right]$ & $\theta_{15}=\frac{M_{d} \bar{X}}{M_{d} \bar{X}+C_{x}}$ & $\frac{(1-f)}{n} \bar{Y}\left(\theta_{15}^{2} C_{x}^{2}-\theta_{15} \rho C_{x} C_{y}\right)$ & $\frac{(1-f)}{n} \bar{Y}^{2}\left(C_{y}^{2}+\theta_{15}^{2} C_{x}^{2}-2 \theta_{15} \rho C_{x} C_{y}\right)$ \\
\hline $\begin{array}{l}\hat{\bar{Y}}_{16}=\bar{y}\left[\frac{C_{x} \bar{X}+M_{d}}{C_{X} \bar{x}+M_{d}}\right] \\
\text { Subrramani and } \\
\text { Kumarapandiyan } \\
\text { (2012c) }\end{array}$ & $\theta_{16}=\frac{C_{x} \bar{X}}{C_{x} \bar{X}+M_{d}}$ & $\frac{(1-f)}{n} \bar{Y}\left(\theta_{16}^{2} C_{x}^{2}-\theta_{16} \rho C_{x} C_{y}\right)$ & $\frac{(1-f)}{n} \bar{Y}^{2}\left(C_{y}^{2}+\theta_{16}^{2} C_{x}^{2}-2 \theta_{16} \rho C_{x} C_{y}\right)$ \\
\hline $\begin{array}{l}\hat{\hat{Y}_{17}}=\bar{y}\left[\frac{\beta_{1} \bar{X}+\beta_{2}}{\beta_{1} \bar{X}+\beta_{2}}\right] \\
\text { Yan and Tian } \\
\text { (2010) }\end{array}$ & $\theta_{17}=\frac{\beta_{1} \bar{X}}{\beta_{1} \bar{X}+\beta_{2}}$ & $\frac{(1-f)}{n} \bar{Y}\left(\theta_{17}^{2} C_{x}^{2}-\theta_{17} \rho C_{x} C_{y}\right)$ & $\frac{(1-f)}{n} \bar{Y}^{2}\left(C_{y}^{2}+\theta_{17}^{2} C_{x}^{2}-2 \theta_{17} \rho C_{x} C_{y}\right)$ \\
\hline
\end{tabular}




\section{MODIFIED RATIO FOR ESTIMATION OF FINITE POPULATION MEAN}

Table 1 Continued

\begin{tabular}{|c|c|c|c|}
\hline Estimator & Constant $\theta_{i}$ & Bias $-B()$. & Mean squared error MSE(.) \\
\hline $\begin{array}{l}\hat{\bar{Y}}_{18}=\bar{y}\left[\frac{\beta_{2} \bar{X}+\beta_{1}}{\beta_{2} \bar{X}+\beta_{1}}\right] \\
\text { Yan and Tian } \\
\text { (2010) }\end{array}$ & $\theta_{18}=\frac{\beta_{2} \bar{X}}{\beta_{2} \bar{X}+\beta_{1}}$ & $\frac{(1-f)}{n} \bar{Y}\left(\theta_{18}^{2} C_{x}^{2}-\theta_{18} \rho C_{x} C_{y}\right)$ & $\frac{(1-f)}{n} \bar{Y}^{2}\left(C_{y}^{2}+\theta_{18}^{2} C_{x}^{2}-2 \theta_{18} \rho C_{x} C_{y}\right)$ \\
\hline$\hat{\bar{Y}}_{19}=\bar{y}\left[\frac{\rho \bar{X}+\beta_{2}}{\rho \bar{X}+\beta_{2}}\right]$ & $\theta_{19}=\frac{\rho \bar{X}}{\rho \bar{X}+\beta_{2}}$ & $\frac{(1-f)}{n} \bar{Y}\left(\theta_{19}^{2} C_{x}^{2}-\theta_{19} \rho C_{x} C_{y}\right)$ & $\frac{(1-f)}{n} \bar{Y}^{2}\left(C_{y}^{2}+\theta_{19}^{2} C_{x}^{2}-2 \theta_{19} \rho C_{x} C_{y}\right)$ \\
\hline$\hat{\bar{Y}}_{20}=\bar{y}\left[\frac{\beta_{2} \bar{X}+\rho}{\beta_{2} \bar{X}+\rho}\right]$ & $\theta_{20}=\frac{\beta_{2} \bar{X}}{\beta_{2} \bar{X}+\rho}$ & $\frac{(1-f)}{n} \bar{Y}\left(\theta_{20}^{2} C_{x}^{2}-\theta_{20} \rho C_{x} C_{y}\right)$ & $\frac{(1-f)}{n} \bar{Y}^{2}\left(C_{y}^{2}+\theta_{20}^{2} C_{x}^{2}-2 \theta_{20} \rho C_{x} C_{y}\right)$ \\
\hline$\hat{\bar{Y}}_{21}=\bar{y}\left[\frac{S_{X} \bar{X}+\beta_{2}}{S_{\bar{x}} \bar{x}+\beta_{2}}\right]$ & $\theta_{21}=\frac{S_{x} \bar{X}}{S_{x} \bar{X}+\beta_{2}}$ & $\frac{(1-f)}{n} \bar{Y}\left(\theta_{21}^{2} C_{x}^{2}-\theta_{21} \rho C_{x} C_{y}\right)$ & $\frac{(1-f)}{n} \bar{Y}^{2}\left(C_{y}^{2}+\theta_{21}^{2} C_{x}^{2}-2 \theta_{21} \rho C_{x} C_{y}\right)$ \\
\hline $\begin{array}{l}\hat{\bar{Y}}_{22}=\bar{y}\left[\frac{\beta_{2} \bar{X}+S_{x}}{\beta_{2} \bar{x}+S_{x}}\right] \\
\operatorname{Singh}(2003)\end{array}$ & $\theta_{22}=\frac{\beta_{2} \bar{X}}{\beta_{2} \bar{X}+S_{x}}$ & $\frac{(1-f)}{n} \bar{Y}\left(\theta_{22}^{2} C_{x}^{2}-\theta_{22} \rho C_{x} C_{y}\right)$ & $\frac{(1-f)}{n} \bar{Y}^{2}\left(C_{y}^{2}+\theta_{22}^{2} C_{x}^{2}-2 \theta_{22} \rho C_{x} C_{y}\right)$ \\
\hline$\hat{\bar{Y}}_{23}=\bar{y}\left[\frac{M_{d} \bar{X}+\beta_{2}}{M_{d} \bar{x}+\beta_{2}}\right]$ & $\theta_{23}=\frac{M_{d} \bar{X}}{M_{d} \bar{X}+\beta_{2}}$ & $\frac{(1-f)}{n} \bar{Y}\left(\theta_{23}^{2} C_{x}^{2}-\theta_{23} \rho C_{x} C_{y}\right)$ & $\frac{(1-f)}{n} \bar{Y}^{2}\left(C_{y}^{2}+\theta_{23}^{2} C_{x}^{2}-2 \theta_{23} \rho C_{x} C_{y}\right)$ \\
\hline $\begin{array}{l}\hat{\bar{Y}}_{24}=\bar{y}\left[\frac{\beta_{2} \bar{X}+M_{d}}{\beta_{2} \bar{x}+M_{d}}\right] \\
\text { Subramani and } \\
\text { Kumarapandiyan } \\
\text { (2012a) }\end{array}$ & $\theta_{24}=\frac{\beta_{2} \bar{X}}{\beta_{2} \bar{X}+M_{d}}$ & $\frac{(1-f)}{n} \bar{Y}\left(\theta_{24}^{2} C_{x}^{2}-\theta_{24} \rho C_{x} C_{y}\right)$ & $\frac{(1-f)}{n} \bar{Y}^{2}\left(C_{y}^{2}+\theta_{24}^{2} C_{x}^{2}-2 \theta_{24} \rho C_{x} C_{y}\right)$ \\
\hline$\hat{\bar{Y}}_{25}=\bar{y}\left[\frac{\rho \bar{X}+\beta_{1}}{\rho \bar{X}+\beta_{1}}\right]$ & $\theta_{25}=\frac{\rho \bar{X}}{\rho \bar{X}+\beta_{1}}$ & $\frac{(1-f)}{n} \bar{Y}\left(\theta_{25}^{2} C_{x}^{2}-\theta_{25} \rho C_{x} C_{y}\right)$ & $\frac{(1-f)}{n} \bar{Y}^{2}\left(C_{y}^{2}+\theta_{25}^{2} C_{x}^{2}-2 \theta_{25} \rho C_{x} C_{y}\right)$ \\
\hline$\hat{\bar{Y}}_{26}=\bar{y}\left[\frac{\beta_{1} \bar{X}+\rho}{\beta_{1} \bar{x}+\rho}\right]$ & $\theta_{26}=\frac{\beta_{1} \bar{X}}{\beta_{1} \bar{X}+\rho}$ & $\frac{(1-f)}{n} \bar{Y}\left(\theta_{26}^{2} C_{x}^{2}-\theta_{26} \rho C_{x} C_{y}\right)$ & $\frac{(1-f)}{n} \bar{Y}^{2}\left(C_{y}^{2}+\theta_{26}^{2} C_{x}^{2}-2 \theta_{26} \rho C_{x} C_{y}\right)$ \\
\hline$\hat{\bar{Y}}_{27}=\bar{y}\left[\frac{S_{x} \bar{X}+\beta_{1}}{S_{x} \bar{x}+\beta_{1}}\right]$ & $\theta_{27}=\frac{S_{x} \bar{X}}{S_{x} \bar{X}+\beta_{1}}$ & $\frac{(1-f)}{n} \bar{Y}\left(\theta_{27}^{2} C_{x}^{2}-\theta_{27} \rho C_{x} C_{y}\right)$ & $\frac{(1-f)}{n} \bar{Y}^{2}\left(C_{y}^{2}+\theta_{27}^{2} C_{x}^{2}-2 \theta_{27} \rho C_{x} C_{y}\right)$ \\
\hline $\begin{array}{l}\hat{\bar{Y}}_{28}=\bar{y}\left[\frac{\beta_{1} \bar{X}+S_{x}}{\beta_{1} \bar{x}+S_{x}}\right] \\
\operatorname{Singh}(2003)\end{array}$ & $\theta_{28}=\frac{\beta_{1} \bar{X}}{\beta_{1} \bar{X}+S_{x}}$ & $\frac{(1-f)}{n} \bar{Y}\left(\theta_{28}^{2} C_{x}^{2}-\theta_{28} \rho C_{x} C_{y}\right)$ & $\frac{(1-f)}{n} \bar{Y}^{2}\left(C_{y}^{2}+\theta_{28}^{2} C_{x}^{2}-2 \theta_{28} \rho C_{x} C_{y}\right)$ \\
\hline$\hat{\bar{Y}}_{29}=\bar{y}\left[\frac{M_{d} \bar{X}+\beta_{1}}{M_{d} \bar{X}+\beta_{1}}\right]$ & $\theta_{29}=\frac{M_{d} \bar{X}}{M_{d} \bar{X}+\beta_{1}}$ & $\frac{(1-f)}{n} \bar{Y}\left(\theta_{29}^{2} C_{x}^{2}-\theta_{29} \rho C_{x} C_{y}\right)$ & $\frac{(1-f)}{n} \bar{Y}^{2}\left(C_{y}^{2}+\theta_{29}^{2} C_{x}^{2}-2 \theta_{29} \rho C_{x} C_{y}\right)$ \\
\hline $\begin{array}{l}\hat{\bar{Y}}_{30}=\bar{y}\left[\frac{\beta_{1} \bar{X}+M_{d}}{\beta_{1} \bar{x}+M_{d}}\right] \\
\text { Subramani and } \\
\text { Kumarapandiyan } \\
\text { (2012b) }\end{array}$ & $\theta_{30}=\frac{\beta_{1} \bar{X}}{\beta_{1} \bar{X}+M_{d}}$ & $\frac{(1-f)}{n} \bar{Y}\left(\theta_{30}^{2} C_{x}^{2}-\theta_{30} \rho C_{x} C_{y}\right)$ & $\frac{(1-f)}{n} \bar{Y}^{2}\left(C_{y}^{2}+\theta_{30}^{2} C_{x}^{2}-2 \theta_{30} \rho C_{x} C_{y}\right)$ \\
\hline
\end{tabular}




\section{JAMBULINGAM SUBRAMANI}

\begin{tabular}{|c|c|c|c|}
\hline Estimator & Constant $\theta_{i}$ & Bias $-B()$. & Mean squared error MSE(.) \\
\hline$\hat{\bar{Y}}_{31}=\bar{y}\left[\frac{S_{x} \bar{X}+\rho}{S_{x} \bar{x}+\rho}\right]$ & $\theta_{31}=\frac{S_{x} \bar{X}}{S_{x} \bar{X}+\rho}$ & $\frac{(1-f)}{n} \bar{Y}\left(\theta_{31}^{2} C_{x}^{2}-\theta_{31} \rho C_{x} C_{y}\right)$ & $\frac{(1-f)}{n} \bar{Y}^{2}\left(C_{y}^{2}+\theta_{31}^{2} C_{x}^{2}-2 \theta_{31} \rho C_{x} C_{y}\right)$ \\
\hline$\hat{\bar{Y}}_{32}=\bar{y}\left[\frac{\rho \bar{X}+S_{x}}{\rho \bar{x}+S_{x}}\right]$ & $\theta_{32}=\frac{\rho \bar{X}}{\rho \bar{X}+S_{x}}$ & $\frac{(1-f)}{n} \bar{Y}\left(\theta_{32}^{2} C_{x}^{2}-\theta_{32} \rho C_{x} C_{y}\right)$ & $\frac{(1-f)}{n} \bar{Y}^{2}\left(C_{y}^{2}+\theta_{32}^{2} C_{x}^{2}-2 \theta_{32} \rho C_{x} C_{y}\right)$ \\
\hline$\hat{Y}_{33}=\bar{y}\left[\frac{M_{d} \bar{X}+\rho}{M_{d} \bar{x}+\rho}\right]$ & $\theta_{33}=\frac{M_{d} \bar{X}}{M_{d} \bar{X}+\rho}$ & $\frac{(1-f)}{n} \bar{Y}\left(\theta_{33}^{2} C_{x}^{2}-\theta_{33} \rho C_{x} C_{y}\right)$ & $\frac{(1-f)}{n} \bar{Y}^{2}\left(C_{y}^{2}+\theta_{33}^{2} C_{x}^{2}-2 \theta_{33} \rho C_{x} C_{y}\right)$ \\
\hline $\begin{array}{l}\hat{\bar{Y}}_{34}=\bar{y}\left[\frac{\rho \bar{X}+M_{d}}{\rho \bar{x}+M_{d}}\right] \\
\text { Subramani and } \\
\text { Kumarapandiyan } \\
\text { (2013b) }\end{array}$ & $\theta_{34}=\frac{\rho \bar{X}}{\rho \bar{X}+M_{d}}$ & $\frac{(1-f)}{n} \bar{Y}\left(\theta_{34}^{2} C_{x}^{2}-\theta_{34} \rho C_{x} C_{y}\right)$ & $\frac{(1-f)}{n} \bar{Y}^{2}\left(C_{y}^{2}+\theta_{34}^{2} C_{x}^{2}-2 \theta_{34} \rho C_{x} C_{y}\right)$ \\
\hline$\hat{\bar{Y}}_{35}=\bar{y}\left[\frac{M_{d} \bar{x}+S_{x}}{M_{d} \bar{x}+S_{x}}\right]$ & $\theta_{35}=\frac{M_{d} \bar{X}}{M_{d} \bar{X}+S_{x}}$ & $\frac{(1-f)}{n} \bar{Y}\left(\theta_{35}^{2} C_{x}^{2}-\theta_{35} \rho C_{x} C_{y}\right)$ & $\frac{(1-f)}{n} \bar{Y}^{2}\left(C_{y}^{2}+\theta_{35}^{2} C_{x}^{2}-2 \theta_{35} \rho C_{x} C_{y}\right)$ \\
\hline$\hat{\bar{Y}}_{36}=\bar{y}\left[\frac{S_{x} \bar{X}+M_{d}}{S_{x} \bar{x}+M_{d}}\right]$ & $\theta_{36}=\frac{S_{x} \bar{X}}{S_{x} \bar{X}+M_{d}}$ & $\frac{(1-f)}{n} \bar{Y}\left(\theta_{36}^{2} C_{x}^{2}-\theta_{36} \rho C_{x} C_{y}\right)$ & $\frac{(1-f)}{n} \bar{Y}^{2}\left(C_{y}^{2}+\theta_{36}^{2} C_{x}^{2}-2 \theta_{36} \rho C_{x} C_{y}\right)$ \\
\hline
\end{tabular}

\section{Proposed generalized ratio estimator}

As stated earlier, the performance of the estimator of the study variable can be improved by using the known population parameters of the auxiliary variable, which are positively correlated with that of study variable.

The proposed generalized modified ratio estimator for estimating the population mean $\bar{Y}$ is defined as:

$$
\hat{\bar{Y}}_{p_{i}}=\bar{y}\left[\frac{\bar{X}+(1+\alpha) \lambda_{i}}{\bar{X}+(1+\alpha) \lambda_{i}}\right] ; i=1,2,3, \ldots, 36
$$

The bias and mean squared error of the proposed estimator $\hat{\bar{Y}}_{p_{i}}$ have been derived (see Appendix A) and are given below:

$$
B\left(\hat{\bar{Y}}_{p_{i}}\right)=\frac{(1-f)}{n} \bar{Y}\left(\theta_{p_{i}}^{2} C_{x}^{2}-\theta_{p_{i}} \rho C_{x} C_{y}\right) ; i=1,2,3, \ldots, 36
$$




$$
\begin{gathered}
\operatorname{MSE}\left(\hat{\bar{Y}}_{p_{i}}\right)=\frac{(1-f)}{n} \bar{Y}^{2}\left(C_{y}^{2}+\theta_{p_{i}}^{2} C_{x}^{2}-2 \theta_{p_{i}} \rho C_{x} C_{y}\right) ; \\
\text { where } \theta_{p_{i}}=\frac{\bar{x}}{\bar{X}+(1-\alpha) \lambda_{i}} ; i=1,2,3, \ldots, 36
\end{gathered}
$$

where $\lambda_{1}=C_{x}, \quad \lambda_{2}=\beta_{2}, \quad \lambda_{3}=\beta_{1}, \quad \lambda_{4}=\rho, \quad \lambda_{5}=S_{x}, \quad \lambda_{6}=M_{d}, \quad \lambda_{7}=C_{x} / \beta_{2}$, $\lambda_{8}=\beta_{2} / C_{x}, \quad \lambda_{9}=C_{x} / \beta_{1}, \quad \lambda_{10}=\beta_{1} / C_{x}, \quad \lambda_{11}=C_{x} / \rho, \lambda_{12}=\rho / C_{x}, \quad \lambda_{13}=C_{x} / S_{x}$, $\lambda_{14}=S_{x} / C_{x}, \quad \lambda_{15}=C_{x} / M_{d}, \quad \lambda_{16}=M_{d} / C_{x}, \quad \lambda_{17}=\beta_{2} / \beta_{1}, \quad \lambda_{18}=\beta_{1} / \beta_{2}$, $\lambda_{19}=\beta_{2} / \rho, \quad \lambda_{20}=\rho / \beta_{2}, \quad \lambda_{21}=\beta_{2} / S_{x}, \quad \lambda_{22}=S_{x} / \beta_{2}, \quad \lambda_{23}=\beta_{2} / M_{d}$, $\lambda_{24}=M_{d} / \beta_{2}, \quad \lambda_{25}=\beta_{1} / \rho, \quad \lambda_{26}=\rho / \beta_{1}, \quad \lambda_{27}=\beta_{1} / S_{x}, \quad \lambda_{28}=S_{x} / \beta_{1}$, $\lambda_{29}=\beta_{1} / M_{d}, \quad \lambda_{30}=M_{d} / \beta_{1}, \quad \lambda_{31}=\rho / S_{x}, \quad \lambda_{32}=S_{x} / \rho, \quad \lambda_{33}=\rho / M_{d}$, $\lambda_{34}=M_{d} / \rho, \lambda_{35}=S_{x} / M_{d}$, and $\lambda_{36}=M_{d} / S_{x}$

\section{Efficiency of the proposed estimator}

The variance of SRSWOR sample mean $\bar{y}_{\text {srs }}$ is given below:

$$
V\left(\bar{y}_{\text {srs }}\right)=\frac{(1-f)}{n} S_{y}^{2}
$$

The bias and mean squared error of the usual ratio estimator $\hat{\bar{Y}}_{R}$ to the first degree of approximation are given below:

$$
\begin{aligned}
& B\left(\hat{\bar{Y}}_{R}\right)=\frac{(1-f)}{n} \bar{Y}\left(C_{x}^{2}-\rho C_{x} C_{y}\right) \\
& \operatorname{MSE}\left(\hat{\bar{Y}}_{R}\right)=\frac{(1-f)}{n} \bar{Y}^{2}\left(C_{y}^{2}+C_{x}^{2}-2 \rho C_{x} C_{y}\right)
\end{aligned}
$$

The bias and the mean squared error of the modified ratio estimators $\hat{\bar{Y}}_{1}$ to $\hat{\bar{Y}}_{36}$ listed in the Table 1 are represented in a single class as given below: 


\section{JAMBULINGAM SUBRAMANI}

$$
\begin{aligned}
& \hat{\overline{Y_{i}}}=\bar{y}\left[\frac{\bar{X}+\lambda_{i}}{\bar{X}+\lambda_{i}}\right] ; i=1,2,3, \ldots, 36 \\
& B\left(\hat{\bar{Y}}_{i}\right)=\frac{(1-f)}{n} \bar{Y}\left(\theta_{i}^{2} C_{x}^{2}-\rho \theta_{i} C_{x} C_{y}\right) ; i=1,2,3, \ldots, 36 \\
& \operatorname{MSE}\left(\hat{\bar{Y}}_{i}\right)=\frac{(1-f)}{n} \bar{Y}^{2}\left(C_{y}^{2}+\theta_{i}^{2} C_{x}^{2}-2 \rho \theta_{i} C_{x} C_{y}\right) \text { where } \theta_{i}=\frac{\bar{X}}{\bar{X}+\lambda_{i}} ; i=1,2,3, \ldots, 36
\end{aligned}
$$

As discussed earlier, the bias, the mean squared error and the constant of the proposed modified ratio estimator $\hat{\bar{Y}}_{p_{i}}$ are given below:

$$
\begin{aligned}
& B\left(\hat{\bar{Y}}_{p_{i}}\right)=\frac{(1-f)}{n} \bar{Y}\left(\theta_{p_{i}}^{2} C_{x}^{2}-\rho \theta_{p_{i}} C_{x} C_{y}\right) ; i=1,2,3, \ldots, 36 \\
& \operatorname{MSE}\left(\hat{\bar{Y}}_{p_{i}}\right)=\frac{(1-f)}{n} \bar{Y}^{2}\left(C_{y}^{2}+\theta_{p_{i}}^{2} C_{x}^{2}-2 \rho \theta_{p_{i}} C_{x} C_{y}\right) \\
& \text { where } \theta_{p_{i}}=\frac{\bar{X}}{\bar{X}+(1+\alpha) \lambda_{i}} ; i=1,2,3, \ldots, 36
\end{aligned}
$$

where $\lambda_{1}=C_{x}, \quad \lambda_{2}=\beta_{2}, \quad \lambda_{3}=\beta_{1}, \quad \lambda_{4}=\rho, \quad \lambda_{5}=S_{x}, \quad \lambda_{6}=M_{d}, \quad \lambda_{7}=C_{x} / \beta_{2}$, $\lambda_{8}=\beta_{2} / C_{x}, \quad \lambda_{9}=C_{x} / \beta_{1}, \quad \lambda_{10}=\beta_{1} / C_{x}, \quad \lambda_{11}=C_{x} / \rho, \quad \lambda_{12}=\rho / C_{x}, \quad \lambda_{13}=C_{x} / S_{x}$, $\lambda_{14}=S_{x} / C_{x}, \quad \lambda_{15}=C_{x} / M_{d}, \quad \lambda_{16}=M_{d} / C_{x}, \quad \lambda_{17}=\beta_{2} / \beta_{1}, \quad \lambda_{18}=\beta_{1} / \beta_{2}$, $\lambda_{19}=\beta_{2} / \rho, \quad \lambda_{20}=\rho / \beta_{2}, \quad \lambda_{21}=\beta_{2} / S_{x}, \quad \lambda_{22}=S_{x} / \beta_{2}, \quad \lambda_{23}=\beta_{2} / M_{d}$, $\lambda_{24}=M_{d} / \beta_{2}, \quad \lambda_{25}=\beta_{1} / \rho, \quad \lambda_{26}=\rho / \beta_{1}, \quad \lambda_{27}=\beta_{1} / S_{x}, \quad \lambda_{28}=S_{x} / \beta_{1}$, $\lambda_{29}=\beta_{1} / M_{d}, \quad \lambda_{30}=M_{d} / \beta_{1}, \quad \lambda_{31}=\rho / S_{x}, \quad \lambda_{32}=S_{x} / \rho, \quad \lambda_{33}=\rho / M_{d}$, $\lambda_{34}=M_{d} / \rho, \lambda_{35}=S_{x} / M_{d}$, and $\lambda_{36}=M_{d} / S_{x}$

From the expressions given in (10) and (13), the conditions (see Appendix B ) for which the proposed estimator $\hat{\bar{Y}}_{p_{i}}$ are more efficient than the simple random sampling without replacement (SRSWOR) sample mean $\bar{y}_{\text {srs }}$ were derived and are:

$$
\operatorname{MSE}\left(\hat{\bar{Y}}_{p_{i}}\right) \leq V\left(\bar{y}_{r}\right) \text { if } \theta_{p_{i}} \leq 2 \rho \frac{C_{y}}{C_{x}}
$$


From the expressions given in (11) and (13), the conditions (see Appendix C) for which the proposed estimators $\hat{\bar{Y}}_{p_{i}}$ are more efficient than the usual ratio estimator $\hat{\bar{Y}}_{R}$ were derived and are:

$$
\operatorname{MSE}\left(\hat{\bar{Y}}_{p_{i}}\right) \leq \operatorname{MSE}\left(\hat{\bar{Y}}_{R}\right) \text { either } \frac{2 \rho C_{y}}{C_{x}}-1 \leq \theta_{p_{i}} \leq 1 \text { (or) } 1 \leq \theta_{p_{i}} \leq \frac{2 \rho C_{y}}{C_{x}}-1
$$

From the expressions given in (12) and (13), the conditions (see Appendix D) for which the proposed estimators $\hat{\bar{Y}}_{p_{j}} ; j=1,2, \ldots, 5$ are more efficient than the existing modified ratio estimators given in Class $1, \bar{Y}_{i} ; i=1,2,3, \ldots, 11$ were derived and are:

$$
\operatorname{MSE}\left(\hat{\bar{Y}}_{p_{j}}\right) \leq \operatorname{MSE}(\hat{\bar{Y}}) \text { either } \frac{2 \rho C_{y}}{C_{x}}-\theta_{i} \leq \theta_{p_{j}} \leq \theta_{i} \text { (or) } \theta_{i} \leq \theta_{p_{j}} \leq \frac{2 \rho C_{y}}{C_{x}}-\theta_{i}
$$

The conditions in terms of $\alpha$ in which proposed estimator $\hat{\bar{Y}}_{p_{i}}$ performs better than the simple random sampling without replacement (SRSWOR) sample mean $\bar{y}_{\text {srs }}$ were obtained and are:

$$
\operatorname{MSE}\left(\hat{\bar{Y}}_{p_{i}}\right) \leq V\left(\bar{y}_{r}\right) \text { if } \alpha_{i} \geq \frac{\bar{X}}{\lambda_{i}}\left[\left(2 \frac{C_{y}}{C_{x}} \rho\right)^{-1}-1-\frac{\lambda_{i}}{\bar{X}}\right]
$$

From the expression given in (15), the range of $\alpha$ in which proposed estimator $\hat{\bar{Y}}_{p_{i}}$ performs better than the usual ratio estimator $\hat{\bar{Y}}_{R}$ is determined and is:

$$
\begin{gathered}
\operatorname{MSE}\left(\hat{\bar{Y}}_{p_{i}}\right) \leq \operatorname{MSE}\left(\hat{\bar{Y}}_{R}\right) \text { either }-1 \leq \alpha_{i} \leq \frac{\bar{X}}{\lambda_{i}}\left[\left(2 \frac{C_{y}}{C_{x}} \rho-1\right)^{-1}-1-\frac{\lambda_{i}}{\bar{X}}\right] \\
\frac{\bar{X}}{\lambda_{i}}\left[\left(2 \frac{C_{y}}{C_{x}} \rho-1\right)^{-1}-1-\frac{\lambda_{i}}{\bar{X}}\right] \leq \alpha_{i} \leq-1 ; i=1,2,3, \ldots, 36
\end{gathered}
$$




\section{JAMBULINGAM SUBRAMANI}

From the expression given in (16), the range of $\alpha$ in which proposed estimator $\hat{\bar{Y}}_{p_{i}}$ performs better than the existing modified ratio estimators listed in Table 1 is:

$$
\begin{gathered}
\operatorname{MSE}\left(\hat{\bar{Y}}_{p_{i}}\right) \leq \operatorname{MSE}\left(\hat{\bar{Y}}_{i}\right) \text { either } 0 \leq \alpha_{i} \leq \frac{\bar{X}}{\lambda_{i}}\left[\left(2 \frac{C_{y}}{C_{x}} \rho-\theta_{i}\right)^{-1}-1-\frac{\lambda_{i}}{\bar{X}}\right] \\
\frac{\bar{X}}{\lambda_{i}}\left[\left(2 \frac{C_{y}}{C_{x}} \rho-\theta_{i}\right)^{-1}-1-\frac{\lambda_{i}}{\bar{X}}\right] \leq \alpha_{i} \leq 0 ; i=1,2,3, \ldots, 36
\end{gathered}
$$

Particular case:

1) At $\alpha_{i}=\frac{\bar{X}}{\lambda_{i}}\left[\left(2 \frac{C_{y}}{C_{x}} \rho\right)^{-1}-1-\frac{\lambda_{i}}{\bar{X}}\right] ; i=1,2,3, \ldots, 36$, the mean squared error of the proposed estimator $\hat{\bar{Y}}_{p_{i}} ; i=1,2,3, \ldots, 36$ equal to the variance of the SRSWOR sample mean $\bar{y}_{\text {srs }}$.

2) At limit point $\alpha_{i}=\frac{\bar{X}}{\lambda_{i}}\left[\left(2 \frac{C_{y}}{C_{x}} \rho-1\right)^{-1}-1-\frac{\lambda_{i}}{\bar{X}}\right]$ or -1 the mean squared error of the proposed estimator $\hat{\bar{Y}}_{p_{i}} ; i=1,2,3, \ldots, 36$ equal to the mean squared error of the usual ratio estimator $\hat{\bar{Y}}_{R}$

3) At limit point $\frac{\bar{X}}{\lambda_{i}}\left[\left(2 \frac{C_{y}}{C_{x}} \rho-\theta_{i}\right)^{-1}-1-\frac{\lambda_{i}}{\bar{X}}\right]$ or 0 the mean squared error of the proposed estimator $\hat{\bar{Y}}_{p_{i}} ; i=1,2,3, \ldots, 36$ the mean squared error of the existing modified ratio estimators $\hat{\overline{Y_{i}}} ; i=1,2,3, \ldots, 36$

4) At $\alpha_{i}=\frac{\bar{X}}{\lambda_{i}}\left[\left(\frac{C_{y}}{C_{x}} \rho\right)^{-1}-1\right]-1 ; i=1,2,3, \ldots, 36$, the means squared error of the proposed estimator $\hat{\bar{Y}}_{p_{i}} ; i=1,2,3, \ldots, 36$ equal to the variance of the usual linear regression estimator $\hat{\bar{Y}}_{1 r}$ 


\section{MODIFIED RATIO FOR ESTIMATION OF FINITE POPULATION MEAN}

\section{Numerical Study}

The performance of the proposed generalized modified ratio estimator is assessed with that of the SRSWOR sample mean, the usual ratio estimator and the existing modified ratio estimators listed in Table 1 for certain natural populations. In this connection, four natural populations for the assessment of the performance of the proposed estimators with that of existing estimators were considered. Population 1 is taken from Singh and Chaudhary (1986) given in page 108; population 2 and population 3 are taken from Singh and Chaudhary (1986) given in page 177; population 4 is taken from Cochran (1977) given in page 152. The population parameters and the constants computed from the above populations are given below in Table 2, whereas the range of $\alpha$ in which proposed estimator performs better than the existing estimators, the constants, the biases and the mean squared errors of the existing and proposed estimators for the above populations are respectively given from the Tables 3 to 8 .

Table 2. Parameters and constants of the population

\begin{tabular}{ccccc}
\hline Parameters & Population 1 & Population 2 & Population 3 & Population 4 \\
\hline$N$ & 70 & 34 & 34 & 49 \\
$n$ & 25 & 20 & 20 & 20 \\
\hline $\bar{Y}$ & 96.7000 & 856.4118 & 85.6412 & 127.7959 \\
$\bar{X}$ & 175.2671 & 208.8824 & 19.9441 & 103.1429 \\
$\rho$ & 0.7293 & 0.4491 & 0.4453 & 0.9817 \\
$S_{y}$ & 60.4714 & 733.1407 & 73.3141 & 123.1212 \\
$C_{y}$ & 0.6254 & 0.8561 & 0.8561 & 0.9634 \\
$S_{X}$ & 140.8572 & 150.5060 & 15.0215 & 104.4051 \\
$C_{X}$ & 0.8037 & 0.7205 & 0.7532 & 1.0122 \\
$\beta_{2}(x)$ & 7.0952 & 0.0974 & 3.7257 & 7.5114 \\
$\beta_{1}(x)$ & 1.9507 & 0.9782 & 1.1823 & 2.2553 \\
$M_{d}$ & 121.5000 & 150.0000 & 14.2500 & 64.0000 \\
\hline
\end{tabular}




\section{JAMBULINGAM SUBRAMANI}

Table 3. Range of $\alpha$ in which proposed estimator performs better than the usual ratio estimator

\begin{tabular}{|c|c|c|c|c|}
\hline \multirow{2}{*}{ Estimator } & \multicolumn{4}{|c|}{$\alpha$ range $\left(\alpha_{L}, \alpha_{u}\right)$} \\
\hline & Population 1 & Population 2 & Population 3 & Population 4 \\
\hline$\hat{\bar{Y}}_{p_{1}}$ & $(-1,1396.1641)$ & $(-1,4023.1475)$ & $(-1,2138.4916)$ & $(-1,14.3885)$ \\
\hline$\hat{\bar{Y}}_{p_{2}}$ & $(-1,157.2620)$ & $(-1,29751.6396)$ & $(-1,431.5268)$ & $(-1,1.0738)$ \\
\hline$\hat{\bar{Y}}_{p_{3}}$ & $(-1,574.6399)$ & $(-1,2963.2549)$ & $(-1,1361.9917)$ & $(-1,5.9068)$ \\
\hline$\hat{\bar{Y}}_{p_{4}}$ & $(-1,1538.6966)$ & $(-1,6454.9931)$ & $(-1,3617.8302)$ & $(-1,14.8665)$ \\
\hline$\hat{\bar{Y}}_{p_{5}}$ & $(-1,6.9719)$ & $(-1,18.2651)$ & $(-1,106.2772)$ & $(-1,-0.8508)$ \\
\hline$\hat{\bar{Y}}_{p_{6}}$ & $(-1,8.2420)$ & $(-1,18.3301)$ & $(-1,112.0853)$ & $(-1,-0.7566)$ \\
\hline$\hat{\bar{Y}}_{p_{7}}$ & $(-1,9912.1584)$ & $(-1,391.1699)$ & $(-1,7970.1040)$ & $(-1,114.5893)$ \\
\hline$\hat{\bar{Y}}_{p_{8}}$ & $(-1,126.1952)$ & $(-1,21436.6645)$ & $(-1,324.7792)$ & $(-1,1.0991)$ \\
\hline$\hat{\bar{Y}}_{p_{9}}$ & $(-1,2724.4479)$ & $(-1,3935.2639)$ & $(-1,2528.5210)$ & $(-1,33.7053)$ \\
\hline$\hat{\bar{Y}}_{p_{10}}$ & $(-1,461.6418)$ & $(-1,2134.8341)$ & $(-1,1025.6054)$ & $(-1,5.9914)$ \\
\hline$\hat{\bar{Y}}_{p_{11}}$ & $(-1,1017.9517)$ & $(-1,1806.3268)$ & $(-1,951.7156)$ & $(-1,14.1076)$ \\
\hline$\hat{\bar{Y}}_{p_{12}}$ & $(-1,1236.4542)$ & $(-1,4650.7357)$ & $(-1,2724.7029)$ & $(-1,15.0607)$ \\
\hline$\hat{\bar{Y}}_{p_{13}}$ & $(-1,196799.6166)$ & $(-1,605657.2149)$ & $(-1,32137.3735)$ & $(-1,1605.6390)$ \\
\hline$\overline{\bar{Y}}_{p_{14}}$ & $(-1,5.4070)$ & $(-1,12.8811)$ & $(-1,79.8012)$ & $(-1,-0.8490)$ \\
\hline$\hat{\bar{Y}}_{p_{15}}$ & $(-1,169754.4325)$ & $(-1,603621.1259)$ & $(-1,30486.7557)$ & $(-1,983.8649)$ \\
\hline$\hat{\bar{Y}}_{p_{16}}$ & $(-1,6.4278)$ & $(-1,12.9279)$ & $(-1,84.1758)$ & $(-1,-0.7536)$ \\
\hline$\hat{\bar{Y}}_{p_{17}}$ & $(-1,307.7217)$ & $(-1,29101.8696)$ & $(-1,510.3764)$ & $(-1,3.6769)$ \\
\hline$\hat{\bar{Y}}_{p_{18}}$ & $(-1,4083.2802)$ & $(-1,287.8790)$ & $(-1,5077.0982)$ & $(-1,50.8800)$ \\
\hline$\hat{\bar{Y}}_{p_{19}}$ & $(-1,114.4205)$ & $(-1,13361.518)$ & $(-1,191.6042)$ & $(-1,1.0359)$ \\
\hline$\hat{\bar{Y}}_{p_{20}}$ & $(-1,10923.4555)$ & $(-1,628.1634)$ & $(-1,13481.6757)$ & $(-1,118.1797)$ \\
\hline$\hat{\bar{Y}}_{p_{21}}$ & $(-1,22291.3463)$ & $(-1,4477948.8100)$ & $(-1,6496.2013)$ & $(-1,215.5109)$ \\
\hline
\end{tabular}




\section{MODIFIED RATIO FOR ESTIMATION OF FINITE POPULATION MEAN}

Table 3 continued.

\begin{tabular}{|c|c|c|c|c|}
\hline \multirow{2}{*}{ Estimator } & \multicolumn{4}{|c|}{$\alpha$ range $\left(\alpha_{L}, \alpha_{u}\right)$} \\
\hline & Population 1 & Population 2 & Population 3 & Population 4 \\
\hline$\hat{\bar{Y}}_{p_{22}}$ & $(-1,55.5623)$ & $(-1,0.8775)$ & $(-1,398.6828)$ & $(-1,0.1207)$ \\
\hline$\hat{\bar{Y}}_{p_{23}}$ & $(-1,19227.8366)$ & $(-1,4462894.9330)$ & $(-1,6162.5069)$ & $(-1,131.7205)$ \\
\hline$\hat{\bar{Y}}_{p_{24}}$ & $(-1,64.5737)$ & $(-1,0.8838)$ & $(-1,420.3218)$ & $(-1,0.8282)$ \\
\hline$\hat{\bar{Y}}_{p_{25}}$ & $(-1,418.8142)$ & $(-1,1330.3074)$ & $(-1,605.9402)$ & $(-1,5.7807)$ \\
\hline$\hat{\bar{Y}}_{p_{26}}$ & $(-1,3002.4862)$ & $(-1,6314.0002)$ & $(-1,4277.5430)$ & $(-1,34.7834)$ \\
\hline$\hat{\bar{Y}}_{p_{27}}$ & $81082.0243)$ & $(-1,446137.0551)$ & $(-1,20473.1799)$ & $(-1,720.1090)$ \\
\hline$\hat{\bar{Y}}_{p_{28}}$ & $(-1,14.5508)$ & $(-1,17.8444)$ & $(-1,125.8339)$ & $(-1,-0.6635)$ \\
\hline$\hat{\bar{Y}}_{p_{29}}$ & $(-1,69939.2477)$ & $(-1,444637.2376)$ & $(-1,19421.6318)$ & $(-1,441.0376)$ \\
\hline$\hat{\bar{Y}}_{p_{30}}$ & $(-1,17.0283)$ & $(-1,17.908)$ & $(-1,132.7007)$ & $(-1,-0.4511)$ \\
\hline$\hat{\bar{Y}}_{p_{31}}$ & $(-1,216876.3557)$ & $(-1,971664.4899)$ & $(-1,54359.2581)$ & $(-1,1655.5450)$ \\
\hline$\hat{\bar{Y}}_{p_{32}}$ & $(-1,4.8139)$ & $(-1,7.6524)$ & $(-1,46.7706)$ & $(-1,-0.8535)$ \\
\hline$\hat{\bar{Y}}_{p_{33}}$ & $(-1,187072.1402)$ & $(-1,968397.9653)$ & $(-1,51567.3306)$ & $(-1,1014.4570)$ \\
\hline$\hat{\bar{Y}}_{p_{34}}$ & $(-1,5.7402)$ & $(-1,7.6816)$ & $(-1,49.3569)$ & $(-1,-0.7611)$ \\
\hline$\hat{\bar{Y}}_{p_{35}}$ & $(-1,967.5869)$ & $(-1,2888.7708)$ & $(-1,1527.7007)$ & $(-1,8.5485)$ \\
\hline$\hat{\bar{Y}}_{p_{36}}$ & $(-1,1300.7996)$ & $(-1,2908.2988)$ & $(-1,1697.7104)$ & $(-1,24.4109)$ \\
\hline
\end{tabular}




\section{JAMBULINGAM SUBRAMANI}

Table 4. Range of $\alpha$ in which proposed estimator performs better than the existing modified ratio estimators

\begin{tabular}{|c|c|c|c|c|}
\hline \multirow{2}{*}{ Estimator } & \multicolumn{4}{|c|}{$\alpha$ range $\left(\alpha_{L}, \alpha_{u}\right)$} \\
\hline & Population 1 & Population 2 & Population 3 & Population 4 \\
\hline$\hat{\bar{Y}}_{p_{1}}$ w.r.t. $\hat{\bar{Y}}_{1}$ & $(0,1343.3398)$ & $(0,3813.2012)$ & $(0,517.1768)$ & $(0,13.0911)$ \\
\hline$\hat{\bar{Y}}_{p_{2}}$ w.r.t. $\hat{\bar{Y}}_{2}$ & $(0,116.3312)$ & $(0,29531.8209)$ & $(0,25.2048)$ & $(-0.0717,0)$ \\
\hline$\hat{\bar{Y}}_{p_{3}}$ w.r.t. $\hat{\bar{Y}}_{3}$ & $(0,524.4732)$ & $(0,2757.1363)$ & $(0,229.5184)$ & $(0,4.6415)$ \\
\hline$\hat{\bar{Y}}_{p_{4}}$ w.r.t. $\hat{\bar{Y}}_{4}$ & $(0,1485.6902)$ & $(0,6240.8577)$ & $(0,1268.9984)$ & $(0,13.5682)$ \\
\hline$\hat{\bar{Y}}_{p_{5}}$ w.r.t. $\hat{\bar{Y}}_{5}$ & $(-0.1011,0)$ & $(0,0.4679)$ & $(0,0.6773)$ & $(-1.2678,0)$ \\
\hline$\hat{\bar{Y}}_{p_{6}}$ w.r.t. $\hat{\bar{Y}}_{6}$ & $(0,0.2071)$ & $(0,0.4777)$ & $(0,0.8631)$ & $(-1.3241,0)$ \\
\hline$\hat{\bar{Y}}_{p_{7}}$ w.r.t. $\hat{\bar{Y}}_{7}$ & $(0,9857.5942)$ & $(0,249.4093)$ & $(0,4332.2435)$ & $(0,113.2681)$ \\
\hline$\hat{\bar{Y}}_{p_{8}}$ w.r.t. $\hat{\bar{Y}}_{8}$ & $(0,87.6545)$ & $(0,21217.4712)$ & $(0,14.0810)$ & $(-0.0482,0)$ \\
\hline$\hat{\bar{Y}}_{p_{9}}$ w.r.t. $\hat{\bar{Y}}_{9}$ & $(0,2670.6504)$ & $(0,3725.5608)$ & $(0,692.7328)$ & $(0,32.3928)$ \\
\hline$\hat{\bar{Y}}_{p_{10}}$ w.r.t. $\hat{\bar{Y}}_{10}$ & $(0,412.5020)$ & $(0,1934.1048)$ & $(0,135.4434)$ & $(0,4.7253)$ \\
\hline$\hat{\bar{Y}}_{p_{11}}$ w.r.t. $\hat{\bar{Y}}_{11}$ & $(0,965.8454)$ & $(0,1608.9539)$ & $(0,117.6363)$ & $(0,12.8106)$ \\
\hline$\hat{\bar{Y}}_{p_{12}}$ w.r.t. $\hat{\bar{Y}}_{12}$ & $(0,1183.8818)$ & $(0,4439.3079)$ & $(0,787.7525)$ & $(0,13.7621)$ \\
\hline$\hat{\bar{Y}}_{p_{13}}$ w.r.t. $\hat{\bar{Y}}_{13}$ & $(0,196744.7709)$ & $(0,605435.8482)$ & $(0,26599.1484)$ & $(0,1604.3148)$ \\
\hline$\hat{\bar{Y}}_{p_{14}}$ w.r.t. $\hat{\bar{Y}}_{14}$ & $(-0.4252,0)$ & $(-0.2370,0)$ & $(-0.0477,0)$ & $(-1.2694,0)$ \\
\hline$\hat{\bar{Y}}_{p_{15}}$ w.r.t. $\hat{\bar{Y}}_{15}$ & $(0,169699.5893)$ & $(0,603399.7594)$ & $(0,24999.7154)$ & $(0,982.5405)$ \\
\hline$\hat{\bar{Y}}_{p_{16}}$ w.r.t. $\hat{\bar{Y}}_{16}$ & $(-0.2210,0)$ & $(-0.2317,0)$ & $(0,0.0582)$ & $(-1.3250,0)$ \\
\hline$\hat{\bar{Y}}_{p_{17}}$ w.r.t. $\hat{\bar{Y}}_{17}$ & $(0,261.0105)$ & $(0,28882.0870)$ & $(0,35.2418)$ & $(0,2.4381)$ \\
\hline$\hat{\bar{Y}}_{p_{18}}$ w.r.t. $\hat{\bar{Y}}_{18}$ & $(0,4029.1336)$ & $(0,162.2788)$ & $(0,2189.8216)$ & $(0,49.5634)$ \\
\hline$\hat{\bar{Y}}_{p_{19}}$ w.r.t. $\hat{\bar{Y}}_{19}$ & $(0,77.0150)$ & $(0,13143.6647)$ & $(0,4.3618)$ & $(-0.1066,0)$ \\
\hline$\hat{\bar{Y}}_{p_{20}}$ w.r.t. $\hat{\bar{Y}}_{20}$ & $(0,10868.8640)$ & $(0,464.1746)$ & $(0,9009.6165)$ & $(0,116.8585)$ \\
\hline$\hat{\bar{Y}}_{p_{21}}$ w.r.t. $\hat{\bar{Y}}_{21}$ & $(0,22236.6179)$ & $(0,4477727.3738)$ & $(0,3199.5909)$ & $(0,214.1880)$ \\
\hline
\end{tabular}




\section{MODIFIED RATIO FOR ESTIMATION OF FINITE POPULATION MEAN}

Table 4 continued.

\begin{tabular}{|c|c|c|c|c|}
\hline \multirow{2}{*}{ Estimator } & \multicolumn{4}{|c|}{$\alpha$ range $\left(\alpha_{L}, \alpha_{u}\right)$} \\
\hline & Population 1 & Population 2 & Population 3 & Population 4 \\
\hline$\hat{\overline{\bar{Y}}}_{p_{22}}$ w.r.t. $\hat{\bar{Y}}_{22}$ & $(0,27.4608)$ & $(-0.9926,0)$ & $(0,21.4756)$ & $(-0.9064,0)$ \\
\hline$\hat{\bar{Y}}_{p_{23}}$ w.r.t. $\hat{\bar{Y}}_{23}$ & $(0,19173.1292)$ & $(0,4462673.4962)$ & $(0,2954.0212)$ & $(0,130.3989)$ \\
\hline$\hat{\bar{Y}}_{p_{24}}$ w.r.t. $\hat{\bar{Y}}_{24}$ & $(0,34.4533)$ & $(-0.9925,0)$ & $(0,23.9023)$ & $(-0.2968,0)$ \\
\hline$\hat{\bar{Y}}_{p_{25}}$ w.r.t. $\hat{\bar{Y}}_{25}$ & $(0,370.1916)$ & $(0,1140.3196)$ & $(0,49.3999)$ & $(0,4.5165)$ \\
\hline$\hat{\bar{Y}}_{p_{26}}$ w.r.t. $\hat{\bar{Y}}_{26}$ & $(0,2948.5920)$ & $(0,6100.0225)$ & $(0,1667.5443)$ & $(0,33.4704)$ \\
\hline$\hat{\bar{Y}}_{p_{27}}$ w.r.t. $\hat{\bar{Y}}_{27}$ & $(0,81027.2001)$ & $(0,445915.7171)$ & $(0,15429.9892)$ & $(0,718.7848)$ \\
\hline$\hat{\bar{Y}}_{p_{28}}$ w.r.t. $\hat{\bar{Y}}_{28}$ & $(0,2.2599)$ & $(0,0.4053)$ & $(0,1.3412)$ & $(-1.3376,0)$ \\
\hline$\hat{\bar{Y}}_{p_{29}}$ w.r.t. $\hat{\bar{Y}}_{29}$ & $(0,69884.4292)$ & $(0,444415.8999)$ & $(0,14444.8014)$ & $(0,439.7137)$ \\
\hline$\hat{\bar{Y}}_{p_{30}}$ w.r.t. $\hat{\bar{Y}}_{30}$ & $(0,3.2704)$ & $(0,0.4146)$ & $(0,1.6000)$ & $(-1.2834,0)$ \\
\hline$\hat{\bar{Y}}_{p_{31}}$ w.r.t. $\hat{\bar{Y}}_{31}$ & $(0,216821.5087)$ & $(0,971443.0928)$ & $(0,48401.3982)$ & $(0,1654.2204)$ \\
\hline$\hat{\bar{Y}}_{p_{32}}$ w.r.t. $\hat{\bar{Y}}_{32}$ & $(-0.5310,0)$ & $(-0.7110,0)$ & $(-0.6684,0)$ & $(-1.2652,0)$ \\
\hline$\hat{\bar{Y}}_{p_{33}}$ w.r.t. $\hat{\bar{Y}}_{33}$ & $(0,187017.2953)$ & $(0,968176.5684)$ & $(0,45644.6094)$ & $(0,1013.1325)$ \\
\hline$\hat{\bar{Y}}_{p_{34}}$ w.r.t. $\hat{\bar{Y}}_{34}$ & $(-0.3616,0)$ & $(-0.7090,0)$ & $(-0.6313,0)$ & $(-1.3225,0)$ \\
\hline$\hat{\bar{Y}}_{p_{35}}$ w.r.t. $\hat{\bar{Y}}_{35}$ & $(0,915.6162)$ & $(0,2683.0193)$ & $(0,283.1533)$ & $(0,7.2673)$ \\
\hline$\hat{\bar{Y}}_{p_{36}}$ w.r.t. $\hat{\bar{Y}}_{36}$ & $(0,1248.1186)$ & $(0,2702.4493)$ & $(0,342.7819)$ & $(0,23.1028)$ \\
\hline
\end{tabular}




\section{JAMBULINGAM SUBRAMANI}

Table 5. Constant, Bias and Mean squared error of the Existing and Proposed estimators for Population 1

\begin{tabular}{|c|c|c|c|c|c|c|c|}
\hline Estimator & $\theta_{i}$ & $\boldsymbol{B}\left(\hat{\overline{\boldsymbol{Y}}}_{(.)}\right)$ & $\operatorname{MSE}\left(\hat{\bar{Y}}_{(\cdot)}\right)$ & $\begin{array}{l}\operatorname{MSE}\left(\hat{\bar{Y}}_{p_{i}}\right) \\
\text { at } \alpha_{L} \& \alpha_{u}\end{array}$ & $\theta_{p_{i}}$ at $\alpha_{a}$ & $\begin{array}{c}\operatorname{Bias}\left(\hat{\bar{Y}}_{p_{i}}\right) \\
\text { at } \alpha_{a}\end{array}$ & $\begin{array}{c}\operatorname{MSE}\left(\hat{\bar{Y}}_{p_{i}}\right) \\
\text { at } \alpha_{a} \\
\end{array}$ \\
\hline $\bar{y}_{s r s}$ & - & - & 94.0466 & 94.0466 & - & - & - \\
\hline$\hat{\bar{Y}}_{R}$ & - & 0.6946 & 73.0773 & 73.0773 & - & - & - \\
\hline$\hat{\bar{Y}}_{p_{1}}$ & 0.9954 & 0.6842 & 72.4673 & 72.4673 & 0.2448 & 0.1269 & 60.1973 \\
\hline$\hat{\bar{Y}}_{p_{2}}$ & 0.9611 & 0.6076 & 68.0853 & 68.0853 & 0.2945 & 0.1291 & 55.5981 \\
\hline$\hat{\bar{Y}}_{p_{3}}$ & 0.9890 & 0.6695 & 71.6173 & 71.6173 & 0.2545 & 0.1279 & 59.2452 \\
\hline$\hat{\bar{Y}}_{p_{4}}$ & 0.9959 & 0.6851 & 72.5232 & 72.5232 & 0.2442 & 0.1268 & 60.2610 \\
\hline$\hat{\bar{Y}}_{p_{5}}$ & 0.5544 & 0.0116 & 44.0518 & 44.0518 & 0.5672 & 0.0003 & 44.0253 \\
\hline$\hat{\bar{Y}}_{p_{6}}$ & 0.5906 & 0.0219 & 44.1080 & 44.1080 & 0.5666 & 0.0009 & 44.0254 \\
\hline$\hat{\bar{Y}}_{p_{7}}$ & 0.9994 & 0.6932 & 72.9906 & 72.9906 & 0.2389 & 0.1261 & 60.7979 \\
\hline$\hat{\bar{Y}}_{p_{8}}$ & 0.9520 & 0.5880 & 66.9919 & 66.9919 & 0.3069 & 0.1285 & 54.5701 \\
\hline$\hat{\bar{Y}}_{p_{9}}$ & 0.9977 & 0.6893 & 72.7631 & 72.7631 & 0.2415 & 0.1264 & 60.5354 \\
\hline$\hat{\bar{Y}}_{p_{10}}$ & 0.9863 & 0.6635 & 71.2712 & 71.2712 & 0.2584 & 0.1283 & 58.8657 \\
\hline$\hat{\bar{Y}}_{p_{11}}$ & 0.9938 & 0.6803 & 72.2439 & 72.2439 & 0.2474 & 0.1272 & 59.9443 \\
\hline$\hat{\bar{Y}}_{p_{12}}$ & 0.9948 & 0.6828 & 72.3894 & 72.3894 & 0.2457 & 0.1270 & 60.1089 \\
\hline$\hat{\bar{Y}}_{p_{13}}$ & 1.0000 & 0.6946 & 73.0729 & 73.0729 & 0.2380 & 0.1260 & 60.8934 \\
\hline$\hat{\bar{Y}}_{p_{14}}$ & 0.5000 & 0.0542 & 44.7328 & 44.7328 & 0.5595 & 0.0072 & 44.0353 \\
\hline$\hat{\bar{Y}}_{p_{15}}$ & 1.0000 & 0.6946 & 73.0722 & 73.0722 & 0.2380 & 0.1260 & 60.8925 \\
\hline$\hat{\bar{Y}}_{p_{16}}$ & 0.5369 & 0.0264 & 44.1707 & 44.1707 & 0.5659 & 0.0015 & 44.0257 \\
\hline$\hat{\bar{Y}}_{p_{17}}$ & 0.9797 & 0.6485 & 70.4101 & 70.4101 & 0.2682 & 0.1289 & 57.9425 \\
\hline$\hat{\bar{Y}}_{p_{18}}$ & 0.9984 & 0.6911 & 72.8672 & 72.8672 & 0.2403 & 0.1263 & 60.6553 \\
\hline$\hat{\bar{Y}}_{p_{19}}$ & 0.9474 & 0.5781 & 66.4416 & 66.4416 & 0.3132 & 0.1279 & 54.0709 \\
\hline
\end{tabular}


MODIFIED RATIO FOR ESTIMATION OF FINITE POPULATION MEAN

Table 5 continued.

\begin{tabular}{|c|c|c|c|c|c|c|c|}
\hline Estimator & $\theta_{i}$ & $\boldsymbol{B}\left(\hat{\overline{\boldsymbol{Y}}}_{(\cdot)}\right)$ & $\operatorname{MSE}\left(\hat{\overline{\mathbf{Y}}}_{(\cdot)}\right)$ & $\begin{array}{l}\operatorname{MSE}\left(\hat{\bar{Y}}_{P_{i}}\right) \\
\text { at } \alpha_{L} \& \alpha_{u}\end{array}$ & $\theta_{p_{i}}$ at $\alpha_{a}$ & $\begin{array}{c}\operatorname{Bias}\left(\hat{\bar{Y}}_{p_{i}}\right) \\
\text { at } \alpha_{a} \\
\end{array}$ & $\begin{array}{c}\operatorname{MSE}\left(\hat{\bar{Y}}_{p_{i}}\right) \\
\text { at } \alpha_{a}\end{array}$ \\
\hline$\hat{\bar{Y}}_{p_{20}}$ & 0.9994 & 0.6933 & 72.9986 & 72.9986 & 0.2388 & 0.1261 & 60.8072 \\
\hline$\hat{\bar{Y}}_{p_{21}}$ & 0.9997 & 0.6940 & 73.0387 & 73.0387 & 0.2383 & 0.1260 & 60.8536 \\
\hline$\hat{\bar{Y}}_{p_{22}}$ & 0.8983 & 0.4772 & 61.0160 & 61.0160 & 0.3747 & 0.1160 & 49.7965 \\
\hline$\hat{\bar{Y}}_{p_{23}}$ & 0.9997 & 0.6939 & 73.0325 & 73.0325 & 0.2384 & 0.1260 & 60.8465 \\
\hline$\hat{\bar{Y}}_{p_{24}}$ & 0.9110 & 0.5026 & 62.3499 & 62.3499 & 0.3596 & 0.1201 & 50.7382 \\
\hline$\hat{\bar{Y}}_{p_{25}}$ & 0.9850 & 0.6604 & 71.0929 & 71.0929 & 0.2604 & 0.1284 & 58.6722 \\
\hline$\hat{\bar{Y}}_{p_{26}}$ & 0.9979 & 0.6898 & 72.7920 & 72.7920 & 0.2411 & 0.1264 & 60.5687 \\
\hline$\hat{\bar{Y}}_{p_{27}}$ & 0.9999 & 0.6945 & 73.0667 & 73.0667 & 0.2380 & 0.1260 & 60.8861 \\
\hline$\hat{\bar{Y}}_{p_{28}}$ & 0.7082 & 0.1601 & 47.1006 & 47.1006 & 0.5326 & 0.0298 & 44.2143 \\
\hline$\hat{\bar{Y}}_{p_{29}}$ & 0.9999 & 0.6944 & 73.0650 & 73.0650 & 0.2380 & 0.1260 & 60.8841 \\
\hline$\hat{\bar{Y}}_{p_{30}}$ & 0.7378 & 0.2018 & 48.5296 & 48.5296 & 0.5164 & 0.0424 & 44.4309 \\
\hline$\hat{\bar{Y}}_{p_{31}}$ & 1.0000 & 0.6946 & 73.0733 & 73.0733 & 0.2379 & 0.1260 & 60.8938 \\
\hline$\hat{\bar{Y}}_{p_{32}}$ & 0.4757 & -0.0701 & 45.3331 & 45.3331 & 0.5527 & 0.0132 & 44.0594 \\
\hline$\hat{\bar{Y}}_{p_{33}}$ & 1.0000 & 0.6946 & 73.0727 & 73.0727 & 0.2380 & 0.1260 & 60.8931 \\
\hline$\hat{\bar{Y}}_{p_{34}}$ & 0.5127 & 0.0451 & 44.4921 & 44.4921 & 0.5622 & 0.0048 & 44.0296 \\
\hline$\hat{\bar{Y}}_{p_{35}}$ & 0.9934 & 0.6796 & 72.2012 & 72.2012 & 0.2478 & 0.1272 & 59.8961 \\
\hline$\hat{\bar{Y}}_{p_{36}}$ & 0.9951 & 0.6834 & 72.4230 & 72.4230 & 0.2453 & 0.1269 & 60.1471 \\
\hline
\end{tabular}




\section{JAMBULINGAM SUBRAMANI}

Table 6. Constant, Bias and Mean squared error of the Existing and Proposed estimators for Population 2

\begin{tabular}{|c|c|c|c|c|c|c|c|}
\hline Estimator & $\theta_{i}$ & $B\left(\hat{\bar{Y}}_{i}\right)$ & $\operatorname{MSE}\left(\hat{\bar{Y}}_{i}\right)$ & $\begin{array}{r}\operatorname{MSE}\left(\hat{\bar{Y}}_{p_{i}}\right) \\
\text { at } \alpha_{L} \& \alpha_{u}\end{array}$ & $\theta_{p_{i}}$ at $\alpha_{a}$ & $\begin{array}{c}\operatorname{Bias}\left(\hat{\bar{Y}}_{p_{i}}\right) \\
\text { at } \alpha_{a} \\
\end{array}$ & $\begin{array}{c}\operatorname{MSE}\left(\hat{\bar{Y}}_{p_{i}}\right) \\
\text { at } \alpha_{a} \\
\end{array}$ \\
\hline $\bar{y}_{s r s}$ & - & - & 11066.0800 & 11066.0800 & - & - & . \\
\hline$\hat{\bar{Y}}_{R}$ & - & 4.2694 & 10539.2700 & 10539.2700 & - & - & - \\
\hline$\hat{\bar{Y}}_{p_{1}}$ & 0.9966 & 4.2233 & 10514.2250 & 10514.2250 & 0.1319 & 0.4851 & 10098.8070 \\
\hline$\hat{\bar{Y}}_{p_{2}}$ & 0.9995 & 4.2631 & 10535.8620 & 10535.8620 & 0.1268 & 0.4721 & 10131.5920 \\
\hline$\hat{\bar{Y}}_{p_{3}}$ & 0.9953 & 4.2070 & 10505.3560 & 10505.3560 & 0.1340 & 0.4903 & 10085.4900 \\
\hline$\hat{\bar{Y}}_{p_{4}}$ & 0.9979 & 4.2406 & 10523.6170 & 10523.6170 & 0.1297 & 0.4795 & 10112.9870 \\
\hline$\hat{\bar{Y}}_{p_{5}}$ & 0.5812 & 0.2533 & 8851.7250 & 8851.7250 & 0.5294 & 0.0206 & 8834.0910 \\
\hline$\hat{\bar{Y}}_{p_{6}}$ & 0.5820 & 0.2581 & 8852.3420 & 8852.3420 & 0.5292 & 0.0213 & 8834.1010 \\
\hline$\hat{\bar{Y}}_{p_{7}}$ & 0.9658 & 3.8212 & 10298.4430 & 10298.4430 & 0.1835 & 0.5881 & 9794.7990 \\
\hline$\hat{\bar{Y}}_{p_{8}}$ & 0.9994 & 4.2607 & 10534.5420 & 10534.5420 & 0.1271 & 0.4729 & 10129.5790 \\
\hline$\hat{\bar{Y}}_{p_{9}}$ & 0.9965 & 4.2223 & 10513.6700 & 10513.6700 & 0.1321 & 0.4854 & 10097.9710 \\
\hline$\hat{\bar{Y}}_{p_{10}}$ & 0.9935 & 4.1831 & 10492.3780 & 10492.3780 & 0.1371 & 0.4977 & 10066.1290 \\
\hline$\hat{\bar{Y}}_{p_{11}}$ & 0.9924 & 4.1676 & 10483.9890 & 10483.9890 & 0.1392 & 0.5024 & 10053.6950 \\
\hline$\hat{\bar{Y}}_{p_{12}}$ & 0.9970 & 4.2295 & 10517.5830 & 10517.5830 & 0.1311 & 0.4831 & 10103.8680 \\
\hline$\hat{\bar{Y}}_{p_{13}}$ & 1.0000 & 4.2691 & 10539.1030 & 10539.1030 & 0.1260 & 0.4701 & 10136.5390 \\
\hline$\hat{\bar{Y}}_{p_{14}}$ & 0.5000 & 0.1538 & 8842.8000 & 8842.8000 & 0.5315 & 0.0103 & 8833.9850 \\
\hline$\hat{\bar{Y}}_{p_{15}}$ & 1.0000 & 4.2691 & 10539.1020 & 10539.1020 & 0.1260 & 0.4701 & 10136.5380 \\
\hline$\hat{\bar{Y}}_{p_{16}}$ & 0.5008 & 0.1502 & 8842.3620 & 8842.3620 & 0.5316 & 0.0098 & 8833.9810 \\
\hline$\hat{\bar{Y}}_{p_{17}}$ & 0.9995 & 4.2630 & 10535.7860 & 10535.7860 & 0.1268 & 0.4721 & 10131.4760 \\
\hline$\hat{\bar{Y}}_{p_{18}}$ & 0.9542 & 3.6732 & 10220.4740 & 10220.4740 & 0.2021 & 0.6133 & 9695.2120 \\
\hline$\hat{\bar{Y}}_{p_{19}}$ & 0.9990 & 4.2555 & 10531.6900 & 10531.6900 & 0.1277 & 0.4746 & 10125.2380 \\
\hline
\end{tabular}




\section{MODIFIED RATIO FOR ESTIMATION OF FINITE POPULATION MEAN}

Table 6 continued

\begin{tabular}{|c|c|c|c|c|c|c|c|}
\hline Estimator & $\theta_{i}$ & $B\left(\hat{\bar{Y}}_{i}\right)$ & $\operatorname{MSE}\left(\hat{\bar{Y}}_{i}\right)$ & $\begin{array}{l}\operatorname{MSE}\left(\hat{\bar{Y}}_{p_{i}}\right) \\
\text { at } \alpha_{L} \& \alpha_{u}\end{array}$ & $\theta_{p_{i}}$ at $\alpha_{a}$ & $\begin{array}{c}\operatorname{Bias}\left(\hat{\bar{Y}}_{p_{i}}\right) \\
\text { at } \alpha_{a}\end{array}$ & $\begin{array}{c}\operatorname{MSE}\left(\hat{\bar{Y}}_{p_{i}}\right) \\
\text { at } \alpha_{a}\end{array}$ \\
\hline$\hat{\bar{Y}}_{p_{20}}$ & 0.9784 & 3.9839 & 10385.0680 & 10385.0680 & 0.1628 & 0.5526 & 9911.8290 \\
\hline$\hat{\bar{Y}}_{p_{21}}$ & 1.0000 & 4.2693 & 10539.2480 & 10539.2480 & 0.1259 & 0.4700 & 10136.7600 \\
\hline$\hat{\bar{Y}}_{p_{22}}$ & 0.1191 & 0.4520 & 10180.5970 & 10180.5970 & 0.2117 & 0.6238 & 9646.3850 \\
\hline$\hat{\bar{Y}}_{p_{23}}$ & 1.0000 & 4.2693 & 10539.2480 & 10539.2480 & 0.1259 & 0.4700 & 10136.7600 \\
\hline$\hat{\bar{Y}}_{p_{24}}$ & 0.1195 & 0.4530 & 10178.2990 & 10178.2990 & 0.2122 & 0.6243 & 9643.6140 \\
\hline$\hat{\bar{Y}}_{p_{25}}$ & 0.9897 & 4.1318 & 10464.6450 & 10464.6450 & 0.1438 & 0.5130 & 10025.2640 \\
\hline$\hat{\bar{Y}}_{p_{26}}$ & 0.9978 & 4.2400 & 10523.2690 & 10523.2690 & 0.1298 & 0.4797 & 10112.4600 \\
\hline$\hat{\bar{Y}}_{p_{27}}$ & 1.0000 & 4.2690 & 10539.0430 & 10539.0430 & 0.1260 & 0.4701 & 10136.4470 \\
\hline$\hat{\bar{Y}}_{p_{28}}$ & 0.5758 & 0.2226 & 8847.9320 & 8847.9320 & 0.5303 & 0.0162 & 8834.0370 \\
\hline$\hat{\bar{Y}}_{p_{29}}$ & 1.0000 & 4.2690 & 10539.0420 & 10539.0420 & 0.1260 & 0.4701 & 10136.4460 \\
\hline$\hat{\bar{Y}}_{p_{30}}$ & 0.5767 & 0.2273 & 8848.4820 & 8848.4820 & 0.5301 & 0.0169 & 8834.0440 \\
\hline$\hat{\bar{Y}}_{p_{31}}$ & 1.0000 & 4.2692 & 10539.1660 & 10539.1660 & 0.1260 & 0.4700 & 10136.6350 \\
\hline$\hat{\bar{Y}}_{p_{32}}$ & 0.3840 & 0.5259 & 9009.4490 & 9009.4490 & 0.4916 & 0.1888 & 8847.7480 \\
\hline$\hat{\bar{Y}}_{p_{33}}$ & 1.0000 & 4.2692 & 10539.1660 & 10539.1660 & 0.1260 & 0.4700 & 10136.6350 \\
\hline$\hat{\bar{Y}}_{p_{34}}$ & 0.3848 & 0.5242 & 9007.5850 & 9007.5850 & 0.4921 & 0.1870 & 8847.4570 \\
\hline$\hat{\bar{Y}}_{p_{35}}$ & 0.9952 & 4.2054 & 10504.4910 & 10504.4910 & 0.1343 & 0.4908 & 10084.1940 \\
\hline$\hat{\bar{Y}}_{p_{36}}$ & 0.9953 & 4.2058 & 10504.7220 & 10504.7220 & 0.1342 & 0.4906 & 10084.5400 \\
\hline
\end{tabular}




\section{JAMBULINGAM SUBRAMANI}

Table 7. Constant, Bias and Mean squared error of the Existing and Proposed estimators for Population 3

\begin{tabular}{|c|c|c|c|c|c|c|c|}
\hline Estimator & $\theta_{i}$ & $B\left(\hat{\bar{Y}}_{i}\right)$ & $\operatorname{MSE}\left(\hat{\bar{Y}}_{i}\right)$ & $\begin{array}{l}\operatorname{MSE}\left(\hat{\bar{Y}}_{p_{i}}\right) \\
\text { at } \alpha_{L} \& \alpha_{u}\end{array}$ & $\theta_{p_{i}}$ at $\alpha_{a}$ & $\begin{array}{c}\operatorname{Bias}\left(\hat{\bar{Y}}_{p_{i}}\right) \\
\text { at } \alpha_{a}\end{array}$ & $\begin{array}{c}\operatorname{MSE}\left(\hat{\bar{Y}}_{p_{i}}\right) \\
\text { at } \alpha_{a}\end{array}$ \\
\hline $\bar{y}_{s r s}$ & - & - & 379.4085 & 379.4085 & - & - & - \\
\hline$\hat{\bar{Y}}_{R}$ & - & 1.6938 & 375.8179 & 375.8179 & - & - & - \\
\hline$\hat{\bar{Y}}_{p_{1}}$ & 0.9636 & 1.5119 & 365.6490 & 365.6490 & 0.0926 & 0.1313 & 354.4061 \\
\hline$\hat{\bar{Y}}_{p_{2}}$ & 0.8426 & 0.9723 & 337.4291 & 337.4291 & 0.2824 & 0.2167 & 318.8736 \\
\hline$\hat{\bar{Y}}_{p_{3}}$ & 0.9440 & 1.4178 & 360.5017 & 360.5017 & 0.1272 & 0.1653 & 346.3464 \\
\hline$\hat{\bar{Y}}_{p_{4}}$ & 0.9782 & 1.5835 & 369.6218 & 369.6218 & 0.0658 & 0.0994 & 361.1082 \\
\hline$\hat{\bar{Y}}_{p_{5}}$ & 0.5704 & 0.1257 & 305.3883 & 305.3883 & 0.4979 & 0.0139 & 304.1944 \\
\hline$\hat{\bar{Y}}_{p_{6}}$ & 0.5833 & 0.1543 & 305.9229 & 305.9229 & 0.4944 & 0.0199 & 304.2154 \\
\hline$\hat{\bar{Y}}_{p_{7}}$ & 0.9900 & 1.6427 & 372.9362 & 372.9362 & 0.0435 & 0.0691 & 367.0206 \\
\hline$\hat{\bar{Y}}_{p_{8}}$ & 0.8013 & 0.8111 & 329.7622 & 329.7622 & 0.3340 & 0.1972 & 312.8772 \\
\hline$\hat{\bar{Y}}_{p_{9}}$ & 0.9690 & 1.5385 & 367.1190 & 367.1190 & 0.0827 & 0.1201 & 356.8370 \\
\hline$\hat{\bar{Y}}_{p_{10}}$ & 0.9270 & 1.3383 & 356.2136 & 356.2136 & 0.1560 & 0.1873 & 340.1698 \\
\hline$\hat{\bar{Y}}_{p_{11}}$ & 0.9218 & 1.3142 & 354.9318 & 354.9318 & 0.1647 & 0.1928 & 338.4184 \\
\hline$\hat{\bar{Y}}_{p_{12}}$ & 0.9712 & 1.5491 & 367.7088 & 367.7088 & 0.0787 & 0.1154 & 357.8285 \\
\hline$\hat{\bar{Y}}_{p_{13}}$ & 0.9975 & 1.6810 & 375.0921 & 375.0921 & 0.0290 & 0.0475 & 371.0234 \\
\hline$\hat{\bar{Y}}_{p_{14}}$ & 0.5000 & 0.0105 & 304.1857 & 304.1857 & 0.5060 & 0.0001 & 304.1748 \\
\hline$\hat{\bar{Y}}_{p_{15}}$ & 0.9974 & 1.6803 & 375.0531 & 375.0531 & 0.0293 & 0.0479 & 370.9498 \\
\hline$\hat{\bar{Y}}_{p_{16}}$ & 0.5132 & 0.0124 & 304.1895 & 304.1895 & 0.5060 & 0.0002 & 304.1748 \\
\hline$\hat{\bar{Y}}_{p_{17}}$ & 0.8636 & 1.0586 & 341.7007 & 341.7007 & 0.2537 & 0.2196 & 322.8924 \\
\hline$\hat{\bar{Y}}_{p_{18}}$ & 0.9843 & 1.6144 & 371.3460 & 371.3460 & 0.0542 & 0.0841 & 364.1476 \\
\hline$\hat{\bar{Y}}_{p_{20}}$ & 0.9940 & 1.6634 & 374.1000 & 374.1000 & 0.0357 & 0.0576 & 369.1661 \\
\hline
\end{tabular}


MODIFIED RATIO FOR ESTIMATION OF FINITE POPULATION MEAN

Table 7 continued

\begin{tabular}{|c|c|c|c|c|c|c|c|}
\hline Estimator & $\theta_{i}$ & $B(\hat{\bar{Y}})$ & $\operatorname{MSE}\left(\hat{\bar{Y}}_{i}\right)$ & $\begin{array}{l}\operatorname{MSE}\left(\hat{\overline{\hat{Y}}}_{p_{i}}\right) \\
\text { at } \alpha_{L} \& \alpha_{u}\end{array}$ & $\theta_{p_{i}}$ at $\alpha_{a}$ & $\begin{array}{c}\operatorname{Bias}\left(\hat{\bar{Y}}_{p_{i}}\right) \\
\text { at } \alpha_{a} \\
\end{array}$ & $\begin{array}{c}\operatorname{MSE}\left(\hat{\overline{\hat{Y}}}_{p_{i}}\right) \\
\text { at } \alpha_{a}\end{array}$ \\
\hline$\hat{\bar{Y}}_{p_{21}}$ & 0.9877 & 1.6314 & 372.2986 & 372.2986 & 0.0478 & 0.0752 & 365.8606 \\
\hline$\hat{\bar{Y}}_{p_{22}}$ & 0.8318 & 0.9292 & 335.3364 & 335.3364 & 0.2965 & 0.2132 & 317.0819 \\
\hline$\hat{\bar{Y}}_{p_{23}}$ & 0.9871 & 1.6281 & 372.1130 & 372.1130 & 0.0491 & 0.0769 & 365.5250 \\
\hline$\hat{\bar{Y}}_{p_{24}}$ & 0.8391 & 0.9582 & 336.7384 & 336.7384 & 0.2871 & 0.2157 & 318.2694 \\
\hline$\hat{\bar{Y}}_{p_{25}}$ & 0.8825 & 1.1392 & 345.7872 & 345.7872 & 0.2262 & 0.2171 & 327.1910 \\
\hline$\hat{\bar{Y}}_{p_{26}}$ & 0.9815 & 1.6000 & 370.5415 & 370.5415 & 0.0597 & 0.0913 & 362.7196 \\
\hline$\hat{\bar{Y}}_{p_{27}}$ & 0.9961 & 1.6737 & 374.6820 & 374.6820 & 0.0318 & 0.0517 & 370.2524 \\
\hline$\hat{\bar{Y}}_{p_{28}}$ & 0.6109 & 0.2194 & 307.3971 & 307.3971 & 0.4844 & 0.0360 & 304.3128 \\
\hline$\hat{\bar{Y}}_{p_{29}}$ & 0.9959 & 1.6726 & 374.6210 & 374.6210 & 0.0322 & 0.0523 & 370.1382 \\
\hline$\hat{\bar{Y}}_{p_{30}}$ & 0.6233 & 0.2505 & 308.2092 & 308.2092 & 0.4790 & 0.0446 & 304.3911 \\
\hline$\hat{\bar{Y}}_{p_{31}}$ & 0.9985 & 1.6862 & 375.3879 & 375.3879 & 0.0270 & 0.0444 & 371.5822 \\
\hline$\hat{\bar{Y}}_{p_{32}}$ & 0.3716 & 0.1715 & 309.4927 & 309.4927 & 0.4703 & 0.0577 & 304.5507 \\
\hline$\hat{\bar{Y}}_{p_{33}}$ & 0.9984 & 1.6858 & 375.3647 & 375.3647 & 0.0272 & 0.0447 & 371.5383 \\
\hline$\hat{\bar{Y}}_{p_{34}}$ & 0.3839 & 0.1609 & 308.5583 & 308.5583 & 0.4766 & 0.0482 & 304.4302 \\
\hline$\hat{\bar{Y}}_{p_{35}}$ & 0.9498 & 1.4452 & 361.9937 & 361.9937 & 0.1172 & 0.1563 & 348.6100 \\
\hline$\hat{\bar{Y}}_{p_{36}}$ & 0.9546 & 1.4682 & 363.2505 & 363.2505 & 0.1087 & 0.1482 & 350.5627 \\
\hline
\end{tabular}




\section{JAMBULINGAM SUBRAMANI}

Table 8. Constant, Bias and Mean squared error of the Existing and Proposed estimators for Population 4

\begin{tabular}{|c|c|c|c|c|c|c|c|}
\hline Estimator & $\theta_{i}$ & $B\left(\hat{\bar{Y}}_{i}\right)$ & $\operatorname{MSE}\left(\hat{\bar{Y}}_{i}\right)$ & $\begin{array}{r}\operatorname{MSE}\left(\hat{\bar{Y}}_{p_{i}}\right) \\
\text { at } \alpha_{L} \& \alpha_{u}\end{array}$ & $\theta_{p_{i}}$ at $\alpha_{a}$ & $\begin{array}{c}\operatorname{Bias}\left(\hat{\bar{Y}}_{p_{i}}\right) \\
\text { at } \alpha_{a} \\
\end{array}$ & $\begin{array}{c}\operatorname{MSE}\left(\hat{\bar{Y}}_{p_{i}}\right) \\
\text { at } \alpha_{a} \\
\end{array}$ \\
\hline $\bar{y}_{s r s}$ & - & - & 448.5780 & 448.5780 & - & - & - \\
\hline$\hat{\bar{Y}}_{R}$ & - & 0.2542 & 18.3619 & 18.3619 & - & - & - \\
\hline$\hat{\bar{Y}}_{p_{1}}$ & 0.9903 & 0.2144 & 17.7773 & 17.7773 & 0.9311 & 0.0121 & 16.2363 \\
\hline$\hat{\bar{Y}}_{p_{2}}$ & 0.9321 & 0.0082 & 16.2333 & 16.2333 & 0.9344 & 0.0000 & 16.2307 \\
\hline$\hat{\bar{Y}}_{p_{3}}$ & 0.9786 & 0.1676 & 17.1984 & 17.1984 & 0.9323 & 0.0076 & 16.2329 \\
\hline$\hat{\bar{Y}}_{p_{4}}$ & 0.9906 & 0.2156 & 17.7934 & 17.7934 & 0.9310 & 0.0122 & 16.2364 \\
\hline$\hat{\bar{Y}}_{p_{5}}$ & 0.4970 & 0.8423 & 110.9857 & 110.9857 & 0.7296 & 0.5790 & 36.9976 \\
\hline$\hat{\bar{Y}}_{p_{6}}$ & 0.6171 & 0.7587 & 66.0867 & 66.0867 & 0.8266 & 0.3451 & 21.9799 \\
\hline$\hat{\bar{Y}}_{p_{7}}$ & 0.9987 & 0.2488 & 18.2780 & 18.2780 & 0.9300 & 0.0159 & 16.2404 \\
\hline$\hat{\bar{Y}}_{p_{8}}$ & 0.9329 & 0.0055 & 16.2319 & 16.2319 & 0.9344 & 0.0000 & 16.2307 \\
\hline$\hat{\bar{Y}}_{p_{9}}$ & 0.9957 & 0.2364 & 18.0897 & 18.0897 & 0.9304 & 0.0145 & 16.2387 \\
\hline$\hat{\bar{Y}}_{p_{10}}$ & 0.9789 & 0.1686 & 17.2095 & 17.2095 & 0.9323 & 0.0076 & 16.2330 \\
\hline$\hat{\bar{Y}}_{p_{11}}$ & 0.9901 & 0.2137 & 17.7674 & 17.7674 & 0.9311 & 0.0120 & 16.2362 \\
\hline$\hat{\bar{Y}}_{p_{12}}$ & 0.9907 & 0.2161 & 17.7996 & 17.7996 & 0.9310 & 0.0122 & 16.2364 \\
\hline$\hat{\bar{Y}}_{p_{13}}$ & 0.9999 & 0.2538 & 18.3558 & 18.3558 & 0.9298 & 0.0165 & 16.2412 \\
\hline$\hat{\bar{Y}}_{p_{14}}$ & 0.5000 & 0.8416 & 109.6730 & 109.6730 & 0.7324 & 0.5732 & 36.4262 \\
\hline$\hat{\bar{Y}}_{p_{15}}$ & 0.9998 & 0.2536 & 18.3520 & 18.3520 & 0.9298 & 0.0165 & 16.2411 \\
\hline$\hat{\bar{Y}}_{p_{16}}$ & 0.6200 & 0.7553 & 65.1890 & 65.1890 & 0.8286 & 0.3397 & 21.7747 \\
\hline$\hat{\bar{Y}}_{p_{17}}$ & 0.9687 & 0.1288 & 16.8141 & 16.8141 & 0.9331 & 0.0046 & 16.2315 \\
\hline$\hat{\bar{Y}}_{p_{18}}$ & 0.9971 & 0.2423 & 18.1775 & 18.1775 & 0.9302 & 0.0152 & 16.2395 \\
\hline$\hat{\bar{Y}}_{p_{19}}$ & 0.9309 & 0.0125 & 16.2366 & 16.2366 & 0.9344 & 0.0000 & 16.2307 \\
\hline
\end{tabular}


MODIFIED RATIO FOR ESTIMATION OF FINITE POPULATION MEAN

Table 8 continued

\begin{tabular}{|c|c|c|c|c|c|c|c|}
\hline Estimator & $\theta_{i}$ & $B\left(\hat{\bar{Y}}_{i}\right)$ & $\operatorname{MSE}\left(\hat{\bar{Y}}_{i}\right)$ & $\begin{array}{l}\operatorname{MSE}\left(\hat{\bar{Y}}_{p_{i}}\right) \\
\text { at } \alpha_{L} \& \alpha_{u}\end{array}$ & $\theta_{p_{i}}$ at $\alpha_{a}$ & $\begin{array}{c}\operatorname{Bias}\left(\hat{\bar{Y}}_{p_{i}}\right) \\
\text { at } \alpha_{a}\end{array}$ & $\begin{array}{c}\operatorname{MSE}\left(\hat{\bar{Y}}_{p_{i}}\right) \\
\text { at } \alpha_{a}\end{array}$ \\
\hline$\hat{\bar{Y}}_{p_{20}}$ & 0.9987 & 0.2490 & 18.2805 & 18.2805 & 0.9300 & 0.0160 & 16.2405 \\
\hline$\hat{\bar{Y}}_{p_{21}}$ & 0.9993 & 0.2513 & 18.3169 & 18.3169 & 0.9299 & 0.0162 & 16.2408 \\
\hline$\hat{\bar{Y}}_{p_{22}}$ & 0.8812 & 0.1815 & 17.6298 & 17.6298 & 0.9314 & 0.0109 & 16.2353 \\
\hline$\hat{\bar{Y}}_{p_{23}}$ & 0.9989 & 0.2495 & 18.2887 & 18.2887 & 0.9299 & 0.0160 & 16.2405 \\
\hline$\hat{\bar{Y}}_{p_{24}}$ & 0.9237 & 0.0383 & 16.2874 & 16.2874 & 0.9343 & 0.0004 & 16.2307 \\
\hline$\hat{\bar{Y}}_{p_{25}}$ & 0.9782 & 0.1661 & 17.1814 & 17.1814 & 0.9323 & 0.0074 & 16.2328 \\
\hline$\hat{\bar{Y}}_{p_{26}}$ & 0.9958 & 0.2369 & 18.0976 & 18.0976 & 0.9304 & 0.0145 & 16.2388 \\
\hline$\hat{\bar{Y}}_{p_{27}}$ & 0.9998 & 0.2533 & 18.3483 & 18.3483 & 0.9298 & 0.0165 & 16.2411 \\
\hline$\hat{\bar{Y}}_{p_{28}}$ & 0.6902 & 0.6531 & 45.7570 & 45.7570 & 0.8706 & 0.2153 & 18.2472 \\
\hline$\hat{\bar{Y}}_{p_{29}}$ & 0.9997 & 0.2528 & 18.3398 & 18.3398 & 0.9298 & 0.0164 & 16.2410 \\
\hline$\hat{\bar{Y}}_{p_{30}}$ & 0.7842 & 0.4563 & 27.3969 & 27.3969 & 0.9103 & 0.0851 & 16.5191 \\
\hline$\hat{\bar{Y}}_{p_{31}}$ & 0.9999 & 0.2538 & 18.3560 & 18.3560 & 0.9298 & 0.0165 & 16.2412 \\
\hline$\hat{\bar{Y}}_{p_{32}}$ & 0.4924 & 0.8433 & 112.9917 & 112.9917 & 0.7253 & 0.5877 & 37.8862 \\
\hline$\hat{\bar{Y}}_{p_{33}}$ & 0.9999 & 0.2536 & 18.3523 & 18.3523 & 0.9298 & 0.0165 & 16.2411 \\
\hline$\hat{\bar{Y}}_{p_{34}}$ & 0.6127 & 0.7637 & 67.4673 & 67.4673 & 0.8237 & 0.3534 & 22.3027 \\
\hline$\hat{\bar{Y}}_{p_{35}}$ & 0.9844 & 0.1909 & 17.4704 & 17.4704 & 0.9317 & 0.0097 & 16.2343 \\
\hline$\hat{\bar{Y}}_{p_{36}}$ & 0.9941 & 0.2299 & 17.9954 & 17.9954 & 0.9306 & 0.0138 & 16.2379 \\
\hline
\end{tabular}

From the values of Table 5-Table 8, it is observed that the bias of the proposed modified ratio estimator $\hat{\bar{Y}}_{p_{j}} ; j=1,2, \ldots, 36$ is less than the bias of the usual ratio estimator and the existing modified ratio estimators $\hat{\bar{Y}}_{i} ; i=1,2,3, \ldots, 36$. Similarly, the mean squared error of the proposed modified ratio estimator $\hat{\bar{Y}}_{p_{j}} ; j=1,2, \ldots, 36$ 


\section{JAMBULINGAM SUBRAMANI}

is less than the variance of SRSWOR sample mean, the mean squared error of the

usual ratio estimator and the existing modified ratio estimators $\hat{\bar{Y}}_{p_{j}} ; j=1,2, \ldots, 36$ for all four populations.

\section{Conclusion}

In this article, a generalized modified ratio estimator has been suggested using the known population parameters of the auxiliary variable. Moreover, many modified ratio estimators have been introduced in this article, and have not been discussed earlier in the literature. The bias and mean squared error of the proposed generalized modified ratio estimator are obtained. Furthermore, the conditions have been derived for which the proposed estimator is more efficient than the existing estimators, and it is shown that the SRSWOR sample mean, the usual ratio estimator, the linear regression and the existing modified ratio estimators are particular cases of the proposed estimator. The performances of the proposed estimator are also assessed for some known populations. It is observed that the bias and the mean squared errors of the proposed estimators are less than the bias and the mean squared error of the existing estimators. Moreover, the proposed estimator will be a generalized modified ratio estimator for estimating the population mean of the study variable using the known population parameters of the auxiliary variable.

\section{Acknowledgements}

The author wishes to record his gratitude and thanks to University Grants Commission (UGC) for the financial assistance through UGC-Major Research Project.

\section{References}

Abdia, Y. Z., \& Shahbaz, M. Q. (2006). A comparative study of generalized ratio and regression estimators with their classical counterparts. Pakistan Journal of Statistics and Operation Research, 2(1), 63-68.

Ahmad, Z., Hanif, M., \& Ahmad, M. (2009). Generalized regression-cumratio estimators for two-phase sampling using multi-auxiliary variables. Pakistan Journal of Statistics, 25(2), 93-106. 


\section{MODIFIED RATIO FOR ESTIMATION OF FINITE POPULATION MEAN}

Al-jararha, J., \& Al-Haj Ebrahem, M. (2012). A ratio estimator under general sampling design. Austrian Journal of Statistics, 41(2), 105-115.

Bhushan, S. (2012). Some efficient sampling strategies based on ratio-type estimator. Electronic Journal of Applied Statistical Analysis, 5(1), 74-88.

Cochran, W. G. (1977). Sampling Techniques. Third Edition, Wiley Eastern Limited.

Dalabehera, M. \& Sahoo, L. N. (1994). Comparison of six almost unbiased ratio estimators. QUESTIIO, 18(3), 369-375.

David, I. P., \& Sukhatme, B .V. (1974). On the bias and mean square error of the ratio estimator. Journal of the American Statistical Association, Theory and Methods Section, 69(346): 464-466.

Goodman, L. A., \& Hartley, H. O. (1958). The precision of unbiased ratiotype estimators. Journal of the American Statistical Association, 53(282): 491508.

Gupta, S., \& Shabbir, J. (2008). On improvement in estimating the population mean in simple random sampling. Journal of Applied Statistics, 35(5), 559-566.

Jhajj, H.S., Sharma, M. K., \& Grover, L.K. (2006). Dual of ratio estimators of finite population mean obtained on using linear transformation to auxiliary variable. Journal of Japan Statistical Society, 36(1), 107-119.

Kadilar, C., \& Cingi, H. (2003). A study on the chain ratio type estimator. Hacettepe Journal of Mathematics and Statistics, Vol. 32, 105-108.

Kadilar, C., \& Cingi, H. (2004). Ratio estimators in simple random sampling. Applied Mathematics and Computation, 151, 893-902.

Kadilar, C., \& Cingi, H. (2006a). An improvement in estimating the population mean by using the correlation co-efficient. Hacettepe Journal of Mathematics and Statistics, 35(1), 103-109.

Kadilar, C., \& Cingi, H. (2006b). Improvement in estimating the population mean in simple random sampling. Applied Mathematics Letters, 19, 75-79.

Khoshnevisan, M., Singh, R., Chauhan, P., Sawan, N., \& Smarandache, F. (2007). A general family of estimators for estimating population mean using known value of some population parameter(s). Far East Journal of Theoretical Statistics, 22, 181-191.

Koyuncu, N., \& Kadilar, C. (2009). Efficient Estimators for the Population Mean. Hacettepe Journal of Mathematics and Statistics, 38(2), 217-225. 


\section{JAMBULINGAM SUBRAMANI}

Kulkarni, S. P. (1978). A note on modified ratio estimator using transformation. Journal of the Indian Society of Agricultural Statistics, 30(2), 125-128.

Murthy, M. N. (1967). Sampling Theory and Methods. Calcutta, India: Statistical Publishing Society.

Naik, V. D., \& Gupta, P. C. (1991): A general class of estimators for estimating population mean using auxiliary information. Metrika, 38, 11-17.

Olkin, I. (1958). Multivariate ratio estimation for finite populations. Biometrika, 45, 154-165.

Pathak, P.K. (1964). On sampling schemes providing unbiased ratio estimators. The Annals of Mathematical Statistics, 35(1), 222-231.

Perri, P. F. (2007). Improved ratio-cum-product type estimators. Statistics in Transition, 8(1), 51-69.

Ray, S. K., \& Sahai, A. (1980). Efficient families of ratio and product-type estimators. Biometrika, 67, 211-215.

Reddy, V. N. (1973). On ratio and product methods of estimation. Sankhya $B$, 35(3), 307-316.

Robinson, J. (1987). Conditioning ratio estimates under simple random sampling. Journal of the American Statistical Association, 82 (399), 826-831.

Sen, A. R. (1993). Some early developments in ratio estimation. Biometrical Journal, 35(1), 3-13.

Shabbir, J., \& Yaab, M. Z. (2003). Improvement over transformed auxiliary variable in estimating the finite populations mean. Biometrical Journal, 45(6), 723-729.

Sharma, B., \& Tailor, R. (2010). A new ratio-cum-dual to ratio estimator of finite population mean in simple random sampling. Global Journal of Science Frontier Research, 10(1), 27-31.

Singh, D., \& Chaudhary, F. S. (1986). Theory and analysis of sample survey designs. New Delhi: New Age International Publisher.

Singh, G. N. (2003). On the improvement of product method of estimation in sample surveys. Journal of the Indian Society of Agricultural Statistics, 56(3), 267-275.

Singh, H. P. \& Espejo, M. R. (2003). On linear regression and ratio-product estimation of a finite population mean. The Statistician, 52, 59-67. 


\section{MODIFIED RATIO FOR ESTIMATION OF FINITE POPULATION MEAN}

Singh, H. P., \& Agnihotri, N. (2008). A general procedure of estimating population mean using auxiliary information in sample surveys. Statistics in Transition, 9(1), 71-87.

Singh, H. P., \& Solanki, R. S. (2012). An alternative procedure for estimating the population mean in simple random sampling. Pakistan Journal of Statistics and Operation Research, 8(2), 213-232.

Singh, H. P., \& Tailor, R. (2003). Use of known correlation co-efficient in estimating the finite population means. Statistics in Transition, 6 (4), 555-560.

Singh, H. P., \& Tailor, R. (2005). Estimation of finite population mean with known co-efficient of variation of an auxiliary. Statistica, anno LXV, n.3, pp 301313.

Singh, H. P., Tailor, R., Singh. S., \& Kim, J. M. (2008). A modified estimator of population mean using power transformation. Statistical Papers, 49, 37-58.

Singh, H. P., Tailor, R., Tailor, R. and Kakran, M. S. (2004): An Improved Estimator of Population Mean Using Power Transformation. Journal of the Indian Society of Agricultural Statistics, 58(2), 223-230.

Sisodia, B. V. S., \& Dwivedi, V. K. (1981). A modified ratio estimator using co-efficient of variation of auxiliary variable. Journal of the Indian Society of Agricultural Statistics, 33(2), 13-18.

Solanki, R. S., Singh, H. P., \& Rathour, A. (2012). An alternative estimator for estimating the finite population mean using auxiliary information in sample surveys. ISRN Probability and Statistics, Article ID 657682, 14 pp.

Srivenkataramana, T. (1980). A dual to ratio estimator in sample surveys. Biometrika, 37, 199-204.

Subramani, J., \& Kumarapandiyan, G. (2012a). Modified ratio estimators using known median and co-efficient of kurtosis. American Journal of Mathematics and Statistics, 2(4), 95-100.

Subramani, J., \& Kumarapandiyan, G. (2012b). Estimation of population mean using known median and co-efficient of skewness. American Journal of Mathematics and Statistics. 2(5), 101-107.

Subramani, J., \& Kumarapandiyan, G. (2012c). Estimation of population mean using co-efficient of variation and median of an auxiliary variable, International Journal of Probability and Statistics, 1(4), 111-118.

Subramani, J., \& Kumarapandiyan, G. (2013a). A new modified ratio estimator for estimation of population mean when median of the auxiliary 


\section{JAMBULINGAM SUBRAMANI}

variable is known. Pakistan Journal of Statistics and Operation Research, 9(2), 137-145.

Subramani, J., \& Kumarapandiyan, G. (2013b). Estimation of Population Mean Using Known Correlation Co-Efficient and Median. Journal of Statistical Theory and Applications (to appear).

Tailor, R., \& Sharma, B. (2009). A modified ratio-cum-product estimator of finite population mean using known coefficient of variation and coefficient of kurtosis. Statistics in Transition-New Series, 10(1), 15-24.

Tin, M. (1965). Comparison of some ratio estimators. Journal of the American Statistical Association, 60, 294-307.

Upadhyaya, L. N., \& Singh, H.P. (1999). Use of transformed auxiliary variable in estimating the finite population mean. Biometrical Journal, V41(5), 627-636.

Yan, Z., \& Tian, B. (2010). Ratio method to the mean estimation using coefficient of skewness of auxiliary variable. ICICA 2010, Part II, CCIS 106, pp. 103-111. 


\section{JAMBULINGAM SUBRAMANI}

\section{Appendix A}

An expression for the bias and mean squared error of the proposed estimators $\hat{\bar{Y}}_{p_{j}} ; i=1,2,3, \ldots, 36$ was derived to first order of approximation with the following notations:

Let us define $e_{0}=\frac{\bar{y}-\bar{Y}}{\bar{Y}}$ and $e_{1}=\frac{\bar{X}-\bar{X}}{\bar{X}}$. Further, $\bar{y}=\bar{Y}\left(1+e_{0}\right)$ and $\bar{X}=\bar{X}\left(1+e_{1}\right)$ and from the definition of $e_{0}$ and $e_{1:}$

$E\left[e_{0}\right]=E\left[e_{1}\right]=0$

$E\left[e_{0}^{2}\right]=\frac{(1-f)}{n} C_{y}^{2}$

$E\left[e_{1}^{2}\right]=\frac{(1-f)}{n} C_{x}^{2}$

$E\left[e_{0} e_{1}\right]=\frac{(1-f)}{n} \rho C_{y} C_{x}$ where $C_{x}=\frac{S_{x}}{\bar{X}}, C_{y}=\frac{S_{y}}{\bar{Y}}$ and $\rho=\frac{S_{x y}}{S_{x} S_{y}}$

The bias of a class of proposed estimators $\hat{\bar{Y}}_{p_{i}} ; i=1,2,3, \ldots, 36$ is derived and is:

$$
\begin{aligned}
& \hat{\bar{Y}}_{p_{i}}=\frac{\bar{y}}{\left(\bar{X}+(1+\alpha) \lambda_{i}\right)}\left(\bar{X}+(1+\alpha) \lambda_{i}\right) ; i=1,2,3, \ldots, 36 \\
& \Rightarrow \hat{\bar{Y}}_{p_{i}}=\frac{\bar{y}}{\left(\bar{X}+e_{1} \bar{X}+(1+\alpha) \lambda_{i}\right)}\left(\bar{X}+(1+\alpha) \lambda_{i}\right) \\
& \Rightarrow \hat{\bar{Y}}_{p_{i}}=\frac{\bar{y}}{\left(\bar{X}+(1+\alpha) \lambda_{i}\right)\left(1+\frac{e_{1} \bar{X}}{\left(\bar{X}+(1+\alpha) \lambda_{i}\right)}\right)}\left(\bar{X}+(1+\alpha) \lambda_{i}\right) \\
& \Rightarrow \hat{\bar{Y}}_{p_{i}}=\frac{\bar{y}}{\left(1+\theta_{p_{i}} e_{1}\right)} \text { where } \theta_{p_{i}}=\frac{\bar{X}}{\bar{X}+(1+\alpha) \lambda_{i}} \\
& \Rightarrow \hat{\bar{Y}}_{p_{i}}=\bar{y}\left(1+\theta_{p_{i}} e_{1}\right)^{-1} \\
& \Rightarrow \hat{\bar{Y}}_{p_{i}}=\bar{y}\left(1-\theta_{p_{i}} e_{1}+\theta_{p_{i}}^{2} e_{1}^{2}-\theta_{p_{i}}^{3} e_{1}^{3}+\ldots\right)
\end{aligned}
$$

Neglecting the terms more than $2^{\text {nd }}$ order, results in 


\section{JAMBULINGAM SUBRAMANI}

$$
\begin{aligned}
& \hat{\bar{Y}}_{p_{i}}=\bar{y}\left(1-\theta_{p_{i}} e_{1}+\theta_{p_{i}}^{2} e_{1}^{2}\right) \\
& \Rightarrow \hat{\bar{Y}}_{p_{i}}=\left(\bar{Y}\left(1+e_{0}\right)\right)\left(1-\theta_{p_{i}} e_{1}+\theta_{p_{i}}^{2} e_{1}^{2}\right) \\
& \Rightarrow \hat{\bar{Y}}_{p_{i}}=\left(\bar{Y}+\bar{Y} e_{0}\right)\left(1-\theta_{p_{i}} e_{1}+\theta_{p_{i}}^{2} e_{1}^{2}\right) \\
& \Rightarrow \hat{\bar{Y}}_{p_{i}}=\bar{Y}+\bar{Y} e_{0}-\bar{Y} \theta_{p_{i}} e_{1}-\bar{Y} \theta_{p_{i}} e_{0} e_{1}+\bar{Y} \theta_{p_{i}}^{2} e_{1}^{2}+\bar{Y} \theta_{p_{i}}^{2} e_{0} e_{1}^{2}
\end{aligned}
$$

Neglecting the terms more than $3^{\text {rd }}$ order, results in

$$
\begin{aligned}
& \hat{\bar{Y}}_{p_{i}}=\bar{Y}+\bar{Y} e_{0}-\bar{Y} \theta_{p_{i}} e_{1}-\bar{Y} \theta_{p_{i}} e_{0} e_{1}+\bar{Y} \theta_{p_{i}}^{2} e_{1}^{2} \\
& \Rightarrow \hat{\bar{Y}}=\bar{Y}=\bar{Y} e_{0}-\bar{Y} \theta_{p_{i}} e_{1}-\bar{Y} \theta_{p_{i}} e_{0} e_{1}+\bar{Y} \theta_{p_{i}}^{2} e_{1}^{2}
\end{aligned}
$$

Taking expectation on both sides, results in

$$
\begin{aligned}
& E\left(\hat{\bar{Y}}_{p_{i}}-\bar{Y}\right)=\bar{Y} E\left(e_{0}\right)-\bar{Y} \theta_{p_{i}} E\left(e_{1}\right)-\bar{Y} \theta_{p_{i}} E\left(e_{0} e_{1}\right)+\bar{Y} \theta_{p_{i}}^{2} E\left(e_{1}^{2}\right) \\
& \Rightarrow \operatorname{Bias}\left(\hat{\bar{Y}}_{p_{i}}\right)=\bar{Y} \theta_{p_{i}}^{2} E\left(e_{1}^{2}\right)-\bar{Y} \theta_{p_{i}} E\left(e_{0} e_{1}\right) \\
& \Rightarrow \operatorname{Bias}\left(\hat{\bar{Y}}_{p_{i}}\right)=\bar{Y} \theta_{p_{i}}^{2} \frac{(1-f)}{n} C_{x}^{2}-\bar{Y} \theta_{p_{i}} \frac{(1-f)}{n} \rho C_{y} C_{x} \\
& \Rightarrow \operatorname{Bias}\left(\hat{\bar{Y}}_{p_{i}}\right)=\frac{(1-f)}{n}\left(\bar{Y} \theta_{p_{i}}^{2} C_{x}^{2}-\bar{Y} \theta_{p_{i}} \rho C_{y} C_{x}\right) \\
& \Rightarrow \operatorname{Bias}\left(\hat{\bar{Y}}_{p_{i}}\right)=\frac{(1-f)}{n} \bar{Y}\left(\theta_{p_{i}}^{2} C_{x}^{2}-\theta_{p_{i}} \rho C_{y} C_{x}\right) \text { where } \theta_{p_{i}}==\frac{\bar{X}}{\bar{X}+(1+\alpha) \lambda_{i}}
\end{aligned}
$$

The mean squared error of the proposed estimator $\hat{\bar{Y}}_{p_{i}} ; i=1,2,3, \ldots, 36$ to first order of approximation is derived and is:

$$
\begin{aligned}
& \hat{\bar{Y}}_{p_{i}}=\frac{\bar{y}}{\left(\bar{X}+(1+\alpha) \lambda_{i}\right)}\left(\bar{X}+(1+\alpha) \lambda_{i}\right) ; i=1,2,3, \ldots, 36 \\
& \Rightarrow \hat{\bar{Y}}_{p_{i}}=\frac{\bar{y}}{\left(\bar{X}+e_{1} \bar{X}+(1+\alpha) \lambda_{i}\right)}\left(\bar{X}+(1+\alpha) \lambda_{i}\right) \\
& \Rightarrow \hat{\bar{Y}}_{p_{i}}=\frac{\bar{y}}{\left(\bar{X}+(1+\alpha) \lambda_{i}\right)\left(1+\frac{e_{1} \bar{X}}{\left(\bar{X}+(1+\alpha) \lambda_{i}\right)}\right)}\left(\bar{X}+(1+\alpha) \lambda_{i}\right) \\
& \Rightarrow \hat{\bar{Y}}_{p_{i}}=\frac{\bar{y}}{\left(1+\theta_{p_{i}} e_{1}\right)} \text { where } \theta_{p_{i}}=\frac{\bar{X}}{\bar{X}+(1+\alpha) \lambda_{i}}
\end{aligned}
$$


$\Rightarrow \hat{\bar{Y}}_{p_{i}}=\bar{y}\left(1+\theta_{p_{i}} e_{1}\right)^{-1}$

$\Rightarrow \hat{\bar{Y}}_{p_{i}}=\bar{y}\left(1-\theta_{p_{i}} e_{1}+\theta_{p_{i}}^{2} e_{1}^{2}-\theta_{p_{i}}^{3} e_{1}^{3}+\ldots\right)$

Neglecting the terms more than $1^{\text {st }}$ order, results in

$\Rightarrow \hat{\bar{Y}}_{p_{i}}=\bar{y}\left(1-\theta_{p_{i}} e_{1}\right)$

$\Rightarrow \hat{\bar{Y}}_{p_{i}}=\left(\bar{Y}\left(1+e_{0}\right)\right)\left(1-\theta_{p_{i}} e_{1}\right)$

$\Rightarrow \hat{\bar{Y}}_{p_{i}}=\left(\bar{Y}+\bar{Y} e_{0}\right)\left(1-\theta_{p_{i}} e_{1}\right)$

$\Rightarrow \hat{\bar{Y}}_{p_{i}}=\bar{Y}+\bar{Y} e_{0}-\bar{Y} \theta_{p_{i}} e_{1}-\bar{Y} \theta_{p_{i}} e_{0} e_{1}$

$\Rightarrow \hat{\bar{Y}}_{p_{i}}-\bar{Y}=\bar{Y} e_{0}-\bar{Y} \theta_{p_{i}} e_{1}-\bar{Y} \theta_{p_{i}} e_{0} e_{1}$

Squaring both sides

$\Rightarrow\left(\hat{\bar{Y}}_{p_{i}}-\bar{Y}\right)^{2}=\left(\bar{Y} e_{0}-\bar{Y} \theta_{p_{i}} e_{1}-\bar{Y} \theta_{p_{i}} e_{0} e_{1}\right)^{2}$

Neglecting the terms more than $2^{\text {nd }}$ order, results in $\left(\hat{\bar{Y}}_{p_{i}}-\bar{Y}\right)^{2}=\bar{Y}^{2} e_{0}^{2}-\bar{Y}^{2} \theta_{p_{i}}^{2} e_{1}^{2}-2 \bar{Y}^{2} \theta_{p_{i}} e_{0} e_{1}$

Taking expectation on both sides results in:

$$
\begin{aligned}
& E\left(\hat{\bar{Y}}_{p_{i}}-\bar{Y}\right)^{2}=E\left(\bar{Y}^{2} e_{0}^{2}\right)+\bar{Y}^{2} \theta_{p_{i}}^{2} E\left(e_{1}^{2}\right)-2 \bar{Y}^{2} \theta_{p_{i}} E\left(e_{0} e_{1}\right) \\
& \Rightarrow \operatorname{MSE}\left(\hat{\bar{Y}}_{p_{i}}\right)=\frac{(1-f)}{n}\left(\bar{Y}^{2} C_{y}^{2}+\bar{Y}^{2} \theta_{p_{i}}^{2} C_{x}^{2}-2 \bar{Y}^{2} \theta_{p_{i}} \rho C_{y} C_{x}\right) ; i=1,2,3, \ldots, 36
\end{aligned}
$$

$$
\text { where } \theta_{p_{i}}=\frac{\bar{X}}{\bar{X}+(1+\alpha) \lambda_{i}}
$$

\section{Appendix B}

The conditions for which proposed estimator $\hat{\bar{Y}}_{p_{i}}$ perform better than the SRSWOR sample mean are derived and are given below:

For $\operatorname{MSE}\left(\hat{\bar{Y}}_{p_{j}}\right) \leq V\left(\bar{y}_{r}\right)$ 


\section{JAMBULINGAM SUBRAMANI}

$$
\begin{aligned}
& \frac{(1-f)}{n} \bar{Y}^{2}\left(C_{y}^{2}+\theta_{p_{i}}^{2} C_{x}^{2}-2 \rho \theta_{p_{i}} C_{x} C_{y}\right) \leq \frac{(1-f)}{n} S_{y}^{2} \\
& \Rightarrow \frac{(1-f)}{n} \bar{Y}^{2}\left(C_{y}^{2}+\theta_{p_{i}}^{2} C_{x}^{2}-2 \rho \theta_{p_{i}} C_{x} C_{y}\right) \leq \frac{(1-f)}{n} \bar{Y}^{2} C_{y}^{2} \\
& \Rightarrow\left(C_{y}^{2}+\theta_{p_{i}}^{2} C_{x}^{2}-2 \rho \theta_{p_{i}} C_{x} C_{y}\right) \leq C_{y}^{2} \\
& \Rightarrow \theta_{p_{i}}^{2} C_{x}^{2}-2 \rho \theta_{p_{i}} C_{x} C_{y} \leq C_{y}^{2} \\
& \Rightarrow \theta_{p_{i}}^{2} C_{x}^{2} \leq 2 \rho \theta_{p_{i}} C_{x} C_{y} \\
& \Rightarrow \theta_{p_{i}} C_{x} \leq 2 \rho C_{y} \\
& \Rightarrow \theta_{p_{i}} \leq 2 \rho \frac{C_{y}}{C_{x}}
\end{aligned}
$$

That is, $\operatorname{MSE}\left(\hat{\bar{Y}}_{p_{i}}\right) \leq V\left(\bar{y}_{r}\right)$ if $\theta_{p_{i}} \leq 2 \rho \frac{C_{y}}{C_{x}}$

\section{Appendix C}

The conditions for which proposed estimator $\hat{\bar{Y}}_{p_{i}}$ perform better than the usual ratio estimator are derived and are given below:

For $\operatorname{MSE}\left(\hat{\bar{Y}}_{p_{j}}\right) \leq \operatorname{MSE}\left(\hat{\bar{Y}}_{R}\right)$

$$
\begin{aligned}
\frac{(1-f)}{n} \bar{Y}^{2}\left(C_{y}^{2}\right. & \left.+\theta_{p_{i}}^{2} C_{x}^{2}-2 \rho \theta_{p_{i}} C_{x} C_{y}\right) \leq \frac{(1-f)}{n} \bar{Y}^{2}\left(C_{y}^{2}+C_{x}^{2}-2 \rho C_{x} C_{y}\right) \\
& \Rightarrow\left(C_{y}^{2}+\theta_{p_{i}}^{2} C_{x}^{2}-2 \rho \theta_{p_{i}} C_{x} C_{y}\right) \leq\left(C_{y}^{2}+C_{x}^{2}-2 \rho C_{x} C_{y}\right) \\
& \Rightarrow \theta_{p_{i}}^{2} C_{x}^{2}-2 \rho \theta_{p_{i}} C_{x} C_{y} \leq C_{x}^{2}-2 \rho C_{x} C_{y} \\
& \Rightarrow \theta_{p_{i}}^{2} C_{x}^{2}-C_{x}^{2}-2 \rho \theta_{p_{i}} C_{x} C_{y}+2 \rho C_{x} C_{y} \leq 0 \\
& \Rightarrow\left(\theta_{p_{i}}^{2}-1\right) C_{x}^{2}-2 \rho C_{x} C_{y}\left(\theta_{p}-1\right) \leq 0 \\
& \Rightarrow\left(\theta_{p_{i}}-1\right)\left(\left(\theta_{p_{i}}+1\right) C_{x}^{2}-2 \rho C_{x} C_{y}\right) \leq 0
\end{aligned}
$$

Condition 1: $\left(\theta_{p_{i}}-1\right) \leq 0$ and $\left(\left(\theta_{p_{i}}+1\right) C_{x}^{2}-2 \rho C_{x} C_{y}\right) \geq 0$ 
MODIFIED RATIO FOR ESTIMATION OF FINITE POPULATION MEAN

$$
\begin{aligned}
& \Rightarrow \theta_{p_{i}} \leq 1 \text { and }\left(\theta_{p_{i}}+1\right) C_{x}^{2} \geq 2 \rho C_{x} C_{y} \\
& \Rightarrow \theta_{p_{i}} \leq 1 \text { and } \theta_{p_{i}} \geq \frac{2 \rho C_{y}}{C_{x}}-1 \\
& \Rightarrow \frac{2 \rho C_{y}}{C_{x}}-1 \leq \theta_{p_{i}} \leq 1
\end{aligned}
$$

Condition 2: $\left(\theta_{p_{i}}-1\right) \geq 0$ and $\left(\left(\theta_{p_{i}}+1\right) C_{x}^{2}-2 \rho C_{x} C_{y}\right) \leq 0$

$$
\begin{aligned}
& \Rightarrow \theta_{p_{i}} \geq 1 \text { and }\left(\theta_{p_{i}}+1\right) C_{x}^{2}-2 \rho C_{x} C_{y} \\
& \Rightarrow \theta_{p_{i}} \geq 1 \text { and } \theta_{p_{i}} \leq \frac{2 \rho C_{y}}{C_{x}}-1 \\
& \Rightarrow 1 \leq \theta_{p_{i}} \leq \frac{2 \rho C_{y}}{C_{x}}-1
\end{aligned}
$$

That is, $\operatorname{MSE}\left(\hat{\bar{Y}}_{p_{i}}\right) \leq \operatorname{MSE}\left(\hat{\bar{Y}}_{R}\right)$ either $\frac{2 \rho C_{y}}{C_{x}}-1 \leq \theta_{p_{i}} \leq 1$ (or) $1 \leq \theta_{p_{i}} \leq \frac{2 \rho C_{y}}{C_{x}}-1$

\section{Appendix D}

The conditions for which proposed estimator $\hat{\bar{Y}}_{p_{i}}$ perform better than the existing modified ratio estimators (Class 1 ) are derived and are given below:

For $\operatorname{MSE}\left(\hat{\bar{Y}}_{p_{j}}\right) \leq \operatorname{MSE}\left(\hat{\bar{Y}}_{i}\right) ; i=1,2,3, \ldots, 36$

$$
\begin{aligned}
\frac{(1-f)}{n} \bar{Y}^{2}\left(C_{y}^{2}\right. & \left.+\theta_{p_{i}}^{2} C_{x}^{2}-2 \rho \theta_{p_{i}} C_{x} C_{y}\right) \leq \frac{(1-f)}{n} \bar{Y}^{2}\left(C_{y}^{2}+\theta_{i}^{2} C_{x}^{2}-2 \rho \theta_{i} C_{x} C_{y}\right) \\
& \Rightarrow\left(C_{y}^{2}+\theta_{p_{i}}^{2} C_{x}^{2}-2 \rho \theta_{p_{i}} C_{x} C_{y}\right) \leq\left(C_{y}^{2}+\theta_{i}^{2} C_{x}^{2}-2 \rho \theta_{i} C_{x} C_{y}\right) \\
& \Rightarrow \theta_{p_{i}}^{2} C_{x}^{2}-2 \rho \theta_{p_{i}} C_{x} C_{y} \leq \theta_{i}^{2} C_{x}^{2}-2 \rho \theta_{i} C_{x} C_{y} \\
& \Rightarrow \theta_{p_{i}}^{2} C_{x}^{2}-\theta_{i}^{2} C_{x}^{2}-2 \rho \theta_{p_{i}} C_{x} C_{y}+2 \rho \theta_{i} C_{x} C_{y} \leq 0 \\
& \Rightarrow\left(\theta_{p_{i}}^{2}-\theta_{i}^{2}\right) C_{x}^{2}-2 \rho \theta_{p_{i}} C_{x} C_{y}\left(\theta_{p_{i}}-\theta_{i}\right) \leq 0 \\
& \Rightarrow\left(\theta_{p_{i}}-\theta_{i}\right)\left(\left(\theta_{p_{i}}+\theta_{i}\right) C_{x}^{2}-2 \rho C_{x} C_{y}\right) \leq 0
\end{aligned}
$$

Condition 1: $\left(\theta_{p_{i}}-\theta_{i}\right) \leq 0$ and $\left(\left(\theta_{p}+\theta_{i}\right) C_{x}^{2}-2 \rho C_{x} C_{y}\right) \geq 0$ 


\section{JAMBULINGAM SUBRAMANI}

$$
\begin{aligned}
& \Rightarrow \theta_{p_{i}} \leq \theta_{i} \text { and }\left(\theta_{p_{i}}+\theta_{i}\right) C_{x}^{2} \geq 2 \rho C_{x} C_{y} \\
& \Rightarrow \theta_{p_{i}} \leq \theta_{i} \text { and } \theta_{p_{i}} \geq \frac{2 \rho C_{y}}{C_{x}}-\theta_{i} \\
& \Rightarrow \frac{2 \rho C_{y}}{C_{x}}-\theta_{i} \leq \theta_{p_{i}} \leq \theta_{i}
\end{aligned}
$$

Condition 2: $\left(\theta_{p_{i}}-\theta_{i}\right) \geq 0$ and $\left(\left(\theta_{p_{i}}+\theta_{i}\right) C_{x}^{2}-2 \rho C_{x} C_{y}\right) \leq 0$

$$
\begin{aligned}
& \Rightarrow \theta_{p_{i}} \geq \theta_{i} \text { and }\left(\theta_{p_{i}}+\theta_{i}\right) C_{x}^{2}-2 \rho C_{x} C_{y} \\
& \Rightarrow \theta_{p_{i}} \geq \theta_{i} \text { and } \theta_{p_{i}} \leq \frac{2 \rho C_{y}}{C_{x}}-\theta_{i} \\
& \Rightarrow \theta_{i} \leq \theta_{p_{i}} \leq \frac{2 \rho C_{y}}{C_{x}}-\theta_{i}
\end{aligned}
$$

That is, $\operatorname{MSE}\left(\hat{\overline{Y_{p_{i}}}}\right) \leq \operatorname{MSE}\left(\hat{\overline{Y_{i}}}\right)$ either

$$
\frac{2 \rho C_{y}}{C_{x}}-\theta_{i} \leq \theta_{p_{i}} \leq \theta_{i} \text { (or) } \theta_{i} \leq \theta_{p_{i}} \leq \frac{2 \rho C_{y}}{C_{x}}-\theta_{i}
$$

